This is a post-peer-review, pre-copyedit version of an article published in Mathematics of Control, Signals, and Systems. The final authenticated version is available online at: http://dx.doi.org/https://doi.org/10.1007/ s00498-019-0236-6

\title{
Periodic Adaptive Stabilization of Rapidly Time-Varying Linear Systems
}

\author{
Joel D. Simard \\ Dept. of Elect. and Comp. Eng. \\ Canada N2L 3G1 \\ (email: jdsimard@uwaterloo.ca) \\ Daniel E. Miller ${ }^{\dagger}$ \\ Dept. of Elect. and Comp. Eng. \\ University of Waterloo, Waterloo, ON \\ Canada N2L 3G1 \\ (email: miller@uwaterloo.ca) \\ (Phone: 519-888-4567 ext. 35215)
}

Christopher Nielsen *

Dept. of Elect. and Comp. Eng. University of Waterloo, Waterloo, ON University of Waterloo, Waterloo, ON

Canada N2L 3G1

(email: cnielsen@uwaterloo.ca)

(Phone: 519-888-4567 ext. 32241)

May 17, 2019

\begin{abstract}
Adaptive control deals with systems that have unknown and/or time-varying parameters. Most techniques are proven for the case in which any time-variation is slow, with results for systems with fast time-variations limited to those for which the time-variation is of a known form or for which the plant has stable zero dynamics. In this paper a new adaptive controller design methodology is proposed in which the time-variation can be rapid and the plant may have unstable-zero dynamics. Under the structural assumptions that the plant is relative degree one and that the plant uncertainty is a single scalar variable, as well as some mild regularity assumptions, it is proven that the closed-loop system is exponentially stable under fast parameter variations with persistent jumps. The proposed controller is nonlinear and periodic, and in each period the parameter is estimated and an appropriate stabilizing control signal is applied.
\end{abstract}

Keywords: Adaptive control, gain scheduling control, time-varying plant, periodic control.

\footnotetext{
${ }^{*}$ Research supported by a grant from the Natural Sciences and Engineering Research Council of Canada.

${ }^{\dagger}$ Research supported by a grant from the Natural Sciences and Engineering Research Council of Canada.
} 


\section{Introduction}

The primary objective of adaptive control is to handle systems with parameters that are uncertain. A classical example of such an adaptive controller is a linear time-invariant (LTI) controller having adjustable parameters. Typically a tuning mechanism is used to modify the controller in such a way that it is suitable for the uncertain plant, which usually results in a nonlinear closed-loop system.

In the 1950's, adaptive control methods were adopted in order to deal with systems for which parameters were both uncertain and time-varying. However, the solution to such a general problem could not be found. Focus shifted to a more modest goal of controlling systems for which parameters were uncertain, but fixed. This simplified scenario was still very difficult and it wasn't until around 1980 that a generalized solution was obtained, e.g., [1], [2], [3]. These controllers typically gave poor transient responses and were not robustly stable in the presence of unmodelled dynamics and bounded disturbances, e.g., [4]. In response, a number of approaches were developed to improve performance. These included Certainty Equivalence approaches, e.g., [5], prerouted logic based switching approach, e.g., [6], [7], and more refined methods such as supervisory and multi-model switching control, e.g. [8], [9], [10], [11], [12].

The study of the adaptive control of time-varying systems has been challenging. With modification, many of the earlier adaptive controllers can handle slow time-variation of plant parameters and/or occasional parameter jumps, e.g., [13], [14], [15], [16], [17], [18]. While there are several general results which deal with unstable zero dynamics under moderate time-variations, e.g., [19], [20], [21], the study of rapid time-variation has been limited, with either the form of the time-variations being known, e.g., [22], [23], or plants with stable zero dynamics (the time-varying counterpart of minimum phase), e.g., [24], [25], [26], [27], [28], [29]. There is a recent result by the last co-author and his graduate student, [30], inspired by the related area of gain scheduling, that can handle arbitrarily fast, but bounded, time-variation. The approach imposes stringent conditions on the observability matrix of the plant, which is used in the estimate of the plant states. In this paper we revisit that approach, with an objective of removing the stringent observability assumptions.

The approach of gain scheduling developed alongside adaptive control. In the gain scheduling problem, a plant whose parameters depend on a variable (the gain scheduling parameter) is considered. This variable is assumed to be measurable, e.g., a plane whose dynamics depend on the altitude. Despite gain scheduled controller design being a classical, and often ad-hoc, approach, it has re-gained interest since the 1990's, e.g., [31], [32], [33]. There are many different design methods for gain scheduling; however, the most common is that of varying the controller coefficients based on the current value of the scheduling variable. An important approach considered is that of converting a nonlinear plant to a linear parameter-varying (LPV) system by either regarding the nonlinearity as the scheduling parameter, or linearizing for a set of operating points regarded

as the scheduling parameter. This preserves well-understood linear design tools and allows the utilization of these tools on difficult nonlinear systems. Several controller design approaches have been developed for this situation, typically resulting in a set of LTI compensators where each controller achieves the desired performance specification for a particular instance of the plant, e.g., [33]. Of significance is the invariant set approach developed in [34], [35], [36], [37], [38], where it is shown that polyhedral Lyapunov functions and associated geometrically intuitive methods can be used for controller synthesis. In particular, in [38] it is shown that under some stringent assumptions (see Section 3.1), a continuous-time gain-scheduled output feedback controller 
can be constructed such that the closed-loop system is exponentially stable under arbitrarily fast time-variations in the parameter.

The purpose of this paper is to develop a nonlinear adaptive output feedback controller that can stabilize a linear system with possibly unstable zero dynamics and arbitrarily fast, but bounded, time variations. This is primarily achieved by extending the work on gain scheduling in [38], but here the scheduling parameter is not available to the controller; the approach builds on the approach presented in [30] and [39]. The main result, Theorem 2, proves that the proposed controller achieves closed-loop exponential stability with a bounded gain on the measurement and process noise. This is a strong form of stability, given that the controller, and therefore the closed-loop system, is nonlinear and time-varying.

The proposed controller utilizes a nonlinear continuous-time filter in tandem with a discretized version of the gain-scheduled output feedback controller in [38]. However, the time-varying parameter is replaced with an estimate generated by a discrete-time parameter estimator inspired by [39], yielding a nonlinear adaptive controller.

As noted above, there are very few general stabilization results within adaptive control for systems with unstable zero dynamics and rapid time-variation. Because of the difficulty of the problem and the lack of general results, in this paper the major structural assumptions imposed are that the plant is single-input single-output and that the unmeasurable time-varying parameter is a scalar; while this may seem restrictive, there are many physical examples where the timevariation is represented by a scalar variable, such as the altitude of a plane, the mass of a missile, and the tank level control problem of [38]. Furthermore, this can be viewed as enabling a first step towards a more general theory for multi-input multi-output systems with multiple unknown parameters.

In Section 2, mathematical preliminaries are presented. In Section 3, the problem of LPV stability is introduced. In Subsection 3.1, a number of crucial definitions and results regarding the stability of LPV systems are discussed and necessary conditions are also stated. In Subsection 3.2, additional standing assumptions are introduced. In Section 4, the proposed controller is expanded upon, and a number of key technical results are proven. Subsection 4.1 provides a brief overview of the controller, and each of Subsections 4.2, 4.3, and 4.4 delve into a specific component of the proposed controller (the filter, the discretized gain-scheduled controller, and the estimator, respectively). In Section 5, it is proven that the proposed controller achieves the desired stability objective. Finally, in Section 6 an illustrative example is provided.

\section{Mathematical Preliminaries}

Let $\mathbb{N}$ denote the set of natural numbers, $\mathbb{R}$ denote the set of real numbers, $\mathbb{R}_{+}$denote the set of non-negative real numbers, and $\mathbb{Z}_{+}$denote the set of non-negative integers. We use both the 1 -norm and the $\infty$-norm to measure the size of a vector $x \in \mathbb{R}^{n}$, defined, respectively as

$$
\|x\|_{1}:=\sum_{i=1}^{n}\left|x_{i}\right|, \quad\|x\|_{\infty}:=\max \left\{\left|x_{1}\right|, \ldots,\left|x_{n}\right|\right\}
$$

we also use the corresponding induced 1-norm and $\infty$-norm of a matrix $A \in \mathbb{R}^{m \times n}$ :

$$
\|A\|_{1}:=\sup _{\|x\|_{1} \neq 0} \frac{\|A x\|_{1}}{\|x\|_{1}}, \quad\|A\|_{\infty}:=\sup _{\|x\|_{\infty} \neq 0} \frac{\|A x\|_{\infty}}{\|x\|_{\infty}} .
$$


Occasionally we will leave the norm of a vector, $x$, or matrix, $A$, undecorated when the specific norm used doesn't impact the analysis or results. When handling noise terms we will frequently use a signal norm to measure size, defined as:

$$
\|w\|_{\infty}:=\sup _{t}\|w(t)\|_{\infty}
$$

For a set $\mathcal{S} \subseteq \mathbb{R}^{m \times n}, P C(\mathcal{S})$ denotes the set of all piecewise continuous functions of the form $f: \mathbb{R}_{+} \rightarrow \mathcal{S}$; we let $P C_{\infty}$ denote the set of all piecewise continuous functions $f \in P C(\mathbb{R})$ for which $\|f\|_{\infty}<\infty$. A function $f: \mathbb{R}_{+} \rightarrow \mathcal{S}$ is doubly piecewise smooth on a closed interval $[a, b] \subset \mathbb{R}$ if there exists a finite set $\left\{t_{i}\right\}$ having

$$
a=t_{1}<t_{2}<\cdots<t_{k}=b
$$

so that on each open interval $\left(t_{i}, t_{i+1}\right), i=1,2, \ldots, k-1, f, \dot{f}$, and $\ddot{f}$ are continuous, bounded, and have finite limits as $t \rightarrow t_{i}$ and $t \rightarrow t_{i+1}$. We say that $f: \mathbb{R}_{+} \rightarrow \mathcal{S}$ is doubly piecewise smooth, denoted $f \in P S^{1}(\mathcal{S})$, if it is doubly piecewise smooth on every finite closed interval in $\mathbb{R}_{+}$. With $T_{0}>0$ and $\delta_{\alpha}>0$, we let $P S^{1}\left(\mathcal{S}, T_{0}, \delta_{\alpha}\right)$ denote the set of $f \in P S^{1}(\mathcal{S})$ for which all discontinuities of $(f, \dot{f}, \ddot{f})$ are at least $T_{0}$ seconds apart and satisfy ess sup $\left\|\left[\begin{array}{l}f \\ \dot{f} \\ \ddot{f}\end{array}\right]\right\|_{\infty} \leq \delta_{\alpha}$.

For a set $\mathcal{F} \subset \mathbb{R}$ of the form

$$
\mathcal{F}:=\left[\underline{f}_{1}, \bar{f}_{1}\right] \cup\left[\underline{f}_{2}, \bar{f}_{2}\right] \cup \cdots \cup\left[\underline{f}_{q}, \bar{f}_{q}\right]
$$

satisfying $\underline{f}_{1}<\bar{f}_{1}<\underline{f}_{2}<\bar{f}_{2}<\cdots<\underline{f}_{q}<\bar{f}_{q}$, we define a projection function $\Pi_{\mathcal{F}}: \mathbb{R} \rightarrow \mathcal{F}$ by

$$
\Pi_{\mathcal{F}}(a):= \begin{cases}a, & \text { if } a \in \mathcal{F} \\ \underline{f}_{1}, & \text { if } a<\underline{f}_{j} ; \\ \bar{f}_{j}, & \text { if } a \in\left(\bar{f}_{j}, \frac{1}{2}\left(\bar{f}_{j}+\underline{f}_{j+1}\right)\right] \text { and } j=1,2, \ldots, q-1 ; \\ \underline{f}_{j+1}, & \text { if } a \in\left(\frac{1}{2}\left(\bar{f}_{j}+\underline{f}_{j+1}\right), \underline{f}_{j+1}\right) \text { and } j=1,2, \ldots, q-1 \\ \bar{f}_{q}, & \text { if } a>\bar{f}_{q} .\end{cases}
$$

We will also take advantage of order notation throughout the analysis. We say that $f: \mathbb{R}_{+} \rightarrow$ $\mathbb{R}^{n \times m}$ is of order $T^{j}$, and write $f=\mathcal{O}\left(T^{j}\right)$, when there exist constants $c>0$ and $T_{1}>0$ so that

$$
\|f(T)\| \leq c T^{j}, T \in\left(0, T_{1}\right) .
$$

Sometimes we have a function which depends not only on $T$, but also on a parameter $\alpha$ lying in a set $\mathcal{A} \subset \mathbb{R}$. Then we say $f=\mathcal{O}\left(T^{j}\right)$ if there exists constants $c>0$ and $T_{1}>0$ so that

$$
\|f(T, \alpha)\| \leq c T^{j}, T \in\left(0, T_{1}\right), \alpha \in \mathcal{A} .
$$

For a set $\mathcal{S} \subseteq \mathbb{R}^{m \times n}$ and a function of the form $f: \mathbb{R}_{+} \rightarrow \mathcal{S}$, with a sampling period $T$ let $f[k]:=f(k T)$ for all $k \in \mathbb{Z}_{+}$. 


\section{Problem Formulation}

We consider a time-varying plant of the form

$$
\begin{aligned}
& \dot{x}(t)=A(\alpha(t)) x(t)+B(\alpha(t)) u(t), \quad x(0)=x_{0} \\
& y(t)=C(\alpha(t)) x(t),
\end{aligned}
$$

where $x(t) \in \mathbb{R}^{n}$ is the plant state, $u(t) \in \mathbb{R}$ is the plant input, and $y(t) \in \mathbb{R}$ is the plant output. The plant parameters $A(\alpha), B(\alpha)$, and $C(\alpha)$ are assumed to be known functions of $\alpha$. The parameter $\alpha(t)$ is unmeasurable ${ }^{1}$ and takes values in a known compact subset $\mathcal{A} \subset \mathbb{R}^{p}$. Since the case of $n=1$ corresponds to a minimum phase plant which is well understood (see [24], [25], [26], [27], [28], and [29]), here we will assume that $n \geq 2$. The following assumption is very natural.

Assumption 1: $(A, B)(\alpha)$ is stabilizable for all $\alpha \in \mathcal{A}$, and $(C, A)(\alpha)$ is detectable for all $\alpha \in \mathcal{A}$.

We want to prove a strong exponential form of closed-loop stability. First we ascertain necessary conditions on $A(\alpha), B(\alpha)$, and $C(\alpha)$ such that this is achievable. This has been studied in great detail in [38] in the simpler case of gain scheduling in which $\alpha$ is measurable; the conditions which are proven to be necessary there must, clearly, also be necessary here.

\subsection{Necessary Conditions}

In [38], Blanchini et al. study the control of (1) when $\alpha$ is measurable. In Proposition 3.1 and Theorem 3.1 of [38] it is, in essence, argued that a strong exponential form of stability ${ }^{2}$ is achievable if, and only if, it is achievable using a so-called LPV controller of the form

$$
\begin{aligned}
& \dot{\bar{z}}(t)=\bar{F}(\alpha(t)) \bar{z}(t)+\bar{G}(\alpha(t)) y(t) \\
& u(t)=\bar{H}(\alpha(t)) \bar{z}(t)+\bar{K}(\alpha(t)) y(t) .
\end{aligned}
$$

If we apply this controller to (1), then in closed-loop we obtain:

$$
\left[\begin{array}{c}
\dot{x}(t) \\
\dot{\bar{z}}(t)
\end{array}\right]=\left[\begin{array}{cc}
A(\alpha(t))+B(\alpha(t)) \bar{K}(\alpha(t)) C(\alpha(t)) & B(\alpha(t)) \bar{H}(\alpha(t)) \\
\bar{G}(\alpha(t)) C(\alpha(t)) & \bar{F}(\alpha(t))
\end{array}\right]\left[\begin{array}{c}
x(t) \\
\bar{z}(t)
\end{array}\right] .
$$

To proceed we introduce some definitions in order to formalize the necessary conditions and subsequent analysis.

Definition 1 (LPV Exponential Stability). The system

$$
\dot{\bar{x}}(t)=\bar{A}(\alpha(t)) \bar{x}(t), \quad \bar{x}\left(t_{0}\right)=\bar{x}_{0}
$$

(or simply $\bar{A}(\alpha)$ ) is said to be LPV exponentially stable if there exist constants $\gamma \geq 1$ and $\lambda>0$ such that for every $t_{0} \in \mathbb{R}, \bar{x}_{0} \in \mathbb{R}^{n}$, and $\alpha \in P C(\mathcal{A})$, the solution of (4) satisfies

$$
\|\bar{x}(t)\| \leq \gamma \mathrm{e}^{-\lambda\left(t-t_{0}\right)}\left\|\bar{x}\left(t_{0}\right)\right\|, \quad \text { for } t \geq t_{0} .
$$

\footnotetext{
${ }^{1}$ The value of $\alpha(t)$ is not available to the control law.

${ }^{2}$ In [38] it is actually argued that an asymptotic form of stability is achievable if, and only if, it is achievable by a controller of the form (2). However, it is easy to prove that the controller (2) asserted to exist by Theorem 3.1 of [38] actually provides exponential stability.
} 
The controller (2) exponentially stabilizes the plant (1) if the corresponding closed-loop system (3) is exponentially stable.

At the heart of the approach of [38] is the use of polyhedral Lyapunov functions and their matrix representations in terms of the 1 -norm and $\infty$-norm. For this reason, we would like to ascertain conditions on a matrix $H(\alpha)$ so that it is not only LPV exponentially stable, but also the value of the constant $\gamma$ in (5) can be taken equal to one when using the appropriate norm. To present the results of [38] on the stabilization of the closed-loop system we need several additional concepts and notation defined therein.

Definition 2 (Class $\left.\mathcal{H}_{1}\right)$. A square matrix $H(\alpha)$ is of class $\mathcal{H}_{1}$ if it is a continuous function of $\alpha$ and if there exists a $\bar{\tau}>0$ such that $\|I+\tau H(\alpha)\|_{1}<1$ for all $\tau \in(0, \bar{\tau})$ and $\alpha \in \mathcal{A}$.

Definition 3 ( Class $\left.^{3} \mathcal{H}_{\infty}\right)$. A square matrix $H(\alpha)$ is of class $\mathcal{H}_{\infty}$ if it is a continuous function of $\alpha$ and if there exists a $\bar{\tau}>0$ such that $\|I+\tau H(\alpha)\|_{\infty}<1$ for all $\tau \in(0, \bar{\tau})$ and $\alpha \in \mathcal{A}$.

Proposition 1. (i) For every matrix $H(\alpha) \in \mathcal{H}_{1}$ there exist $\bar{\lambda}<0$ and $\bar{T}>0$ such that for all $\lambda \in(\bar{\lambda}, 0)$ and $T \in(0, \bar{T})$, the following holds:

$$
\|I+T H(\alpha)\|_{1} \leq 1+\lambda T, \quad \alpha \in \mathcal{A} .
$$

(ii) For every matrix $H(\alpha) \in \mathcal{H}_{\infty}$ there exist $\bar{\lambda}<0$ and $\bar{T}>0$ such that for all $\lambda \in(\bar{\lambda}, 0)$ and $T \in(0, \bar{T})$, the following holds:

$$
\|I+T H(\alpha)\|_{\infty} \leq 1+\lambda T, \quad \alpha \in \mathcal{A} .
$$

Proof. The proof of $(i)$ is given in the proof of [40, Proposition 1]. Part $(i i)$ follows from part $(i)$ on observing that $H(\alpha) \in \mathcal{H}_{\infty} \Longleftrightarrow H(\alpha)^{\top} \in \mathcal{H}_{1}$ and $\|I+T H(\alpha)\|_{\infty}=\left\|I+T H(\alpha)^{\top}\right\|_{1}$.

The next proposition elucidates the connection between class $\mathcal{H}_{1}$ and class $\mathcal{H}_{\infty}$ matrices and their strong stability properties.

Proposition 2. If $H(\alpha) \in \mathcal{H}_{1}$ or $H(\alpha) \in \mathcal{H}_{\infty}$, then $H(\alpha)$ is LPV exponentially stable; moreover, if $H(\alpha) \in \mathcal{H}_{1}$ (respectively $H(\alpha) \in \mathcal{H}_{\infty}$ ) and the 1-norm (respectively $\infty$-norm) is used in $(1)$, then $\gamma$ can be chosen to be one.

Proof. The proof for the case of $H(\alpha) \in \mathcal{H}_{1}$ is given in the proof of [40, Proposition 3]. The case of $H(\alpha) \in \mathcal{H}_{\infty}$ follows from a slightly modified argument and can also be found in the proof of [40, Proposition 3].

Now we turn to a key result of [38]: the first part of the result is a restatement of part of Theorem 3.1 of [38]; the second part follows from the details of its proof.

\footnotetext{
${ }^{3}$ This definition of $\mathcal{H}_{\infty}$ is not to be confused with the Hardy space of the same name.
} 
Theorem 1. The system (1) is LPV exponentially stabilizable via an output feedback controller of the form (2) if, and only if, there exists a matrix $P(\alpha) \in \mathcal{H}_{1}$, a matrix $Q(\alpha) \in \mathcal{H}_{\infty}$, a full row-rank matrix $X$, a full column-rank matrix $R$, a row vector $U(\alpha)$, and a column vector $L(\alpha)$ such that the equations

$$
\begin{aligned}
A(\alpha) X+B(\alpha) U(\alpha) & =X P(\alpha) \\
R A(\alpha)+L(\alpha) C(\alpha) & =Q(\alpha) R
\end{aligned}
$$

are satisfied for all $\alpha \in \mathcal{A}$; indeed, with $M$ any left inverse of $R, Z$ chosen so that $\left[\begin{array}{c}X \\ Z\end{array}\right]$ is square and invertible and $V(\alpha):=Z P(\alpha)$, a stabilizing controller of the form (2) can be chosen in the following way: first define

$$
\left[\begin{array}{ll}
K(\alpha) & H(\alpha) \\
G(\alpha) & F(\alpha)
\end{array}\right]:=\left[\begin{array}{l}
U(\alpha) \\
V(\alpha)
\end{array}\right]\left[\begin{array}{l}
X \\
Z
\end{array}\right]^{-1}
$$

and from this we obtain the controller

$$
\begin{gathered}
\dot{\bar{z}}(t):=\left[\begin{array}{c}
\dot{z}(t) \\
\dot{r}(t)
\end{array}\right]=\left[\begin{array}{cc}
F(\alpha) & G(\alpha) M \\
R B(\alpha) H(\alpha) & Q(\alpha)+R B(\alpha) K(\alpha) M
\end{array}\right]\left[\begin{array}{l}
z(t) \\
r(t)
\end{array}\right]+\left[\begin{array}{c}
0 \\
-L(\alpha)
\end{array}\right] y(t), \\
u(t)=\left[\begin{array}{ll}
H(\alpha) & K(\alpha) M
\end{array}\right]\left[\begin{array}{l}
z(t) \\
r(t)
\end{array}\right]
\end{gathered}
$$

it turns out that

$$
\left[\begin{array}{cc}
A(\alpha)+B(\alpha) K(\alpha) & B(\alpha) H(\alpha) \\
G(\alpha) & F(\alpha)
\end{array}\right]=\left[\begin{array}{c}
X \\
Z
\end{array}\right] P(\alpha)\left[\begin{array}{c}
X \\
Z
\end{array}\right]^{-1}
$$

In light of Theorem 1, we impose the following assumption.

Assumption 2: There exists a known matrix $P(\alpha) \in \mathcal{H}_{1}$, a known matrix $Q(\alpha) \in \mathcal{H}_{\infty}$, a known full row-rank matrix $X$, a known full column-rank matrix $R$, a known row vector $U(\alpha)$, and a known column vector $L(\alpha)$ such that (8)-(9) hold for all $\alpha \in \mathcal{A}$.

At this point $Z$ is fixed so that $\left[\begin{array}{c}X \\ Z\end{array}\right]$ is non-singular, and we fix a matrix $M$ to be any left inverse of $R$.

\subsection{Additional Assumptions}

From the previous section we see that stabilizing a time-varying system is difficult, even when the free parameter is known. The difficulty arises in a very subtle way from the existence of unstable zero dynamics, since it is well known that if the zero dynamics are stable then stabilizing in the face of rapid time-variation is possible, e.g., see [25], [26], [27], [28], [29]. Because of the difficulty of the problem and the lack of general results, in this paper we impose a major structural assump- 
tion: we allow one degree of freedom in $\alpha$-it is a scalar.

Assumption 3: $\mathcal{A}$ is a known compact subset of $\mathbb{R}$, consisting of a finite set of closed intervals.

The assumption that $\alpha$ be scalar is reasonable in many situations, e.g., when compensating for altitude in the control of an airplane, or when taking into account the significant loss of mass of a missile during its flight.

We will estimate $\alpha$ using ideas from work by the last co-author [39]. We assume that it can be obtained, roughly speaking, from the plant's first Markov parameter. To this end, define

$$
\begin{aligned}
f: \mathcal{A} & \rightarrow \mathbb{R} \\
\alpha & \mapsto C(\alpha) B(\alpha),
\end{aligned}
$$

as well as the image of $\mathcal{A}$ under $f$ :

$$
\mathcal{F}:=f(\mathcal{A}) .
$$

At this point we impose the second major structural assumption.

Assumption 4: The function $f: \mathcal{A} \rightarrow \mathcal{F}$ is one-to-one and its inverse $f^{-1}$ is Lipschitz continuous on $\mathcal{F}$.

Now we turn to more routine regularity assumptions needed to prove that our approach will work. First of all, we assume that $A, B$, and $C$ are well-behaved as functions of the parameter $\alpha$.

Assumption 5: $A(\alpha), B(\alpha), C(\alpha)$, and $\frac{\mathrm{d} C(\alpha)}{\mathrm{d} \alpha}$ are Lipschitz continuous on $\mathcal{A}$.

We impose similar conditions on $P(\alpha)$ and $Q(\alpha)$ of Assumption 2:

Assumption 6: $P(\alpha), Q(\alpha), U(\alpha)$ and $L(\alpha)$ are Lipschitz continuous on $\mathcal{A}$.

Remark 1. It follows from Theorem 1 that the corresponding controller matrices $F(\alpha), G(\alpha)$, $H(\alpha)$, and $K(\alpha)$ are also Lipschitz continuous on $\mathcal{A}$.

While seemingly restrictive, there exist physical systems satisfying all of Assumptions 1 to 6 , as the next example illustrates.

Example 1. Consider an inverted pendulum with a fixed pivot, with the angle $\theta$ being measured from the upward vertical position of the pendulum. The pendulum has a mass $m$ located a timevarying distance $l(t)$ away from the pivot. Taking the input to be an applied torque $u$, the output to be the velocity of the pendulum $\dot{\theta}$, and restricting $l(t) \in[a, b]$ for some $0<a<b$, this yields the model

$$
\begin{aligned}
\ddot{\theta}(t) & =\frac{g}{l(t)} \sin (\theta(t))+\frac{u(t)}{m l(t)^{2}}, \\
y(t) & =\dot{\theta}(t) .
\end{aligned}
$$


Linearizing the system at the upright position yields the linear system

$$
\begin{aligned}
{\left[\begin{array}{c}
\dot{\theta}(t) \\
\ddot{\theta}(t)
\end{array}\right] } & =\left[\begin{array}{cc}
0 & 1 \\
\frac{g}{l(t)} & 0
\end{array}\right]\left[\begin{array}{c}
\theta(t) \\
\dot{\theta}(t)
\end{array}\right]+\left[\begin{array}{c}
0 \\
\frac{1}{m l(t)^{2}}
\end{array}\right] u(t), \\
y(t) & =\left[\begin{array}{ll}
0 & 1
\end{array}\right]\left[\begin{array}{c}
\theta(t) \\
\dot{\theta}(t)
\end{array}\right] .
\end{aligned}
$$

Since there is only one time-varying parameter, Assumption 3 holds with $\mathcal{A}=[a, b]$. Moreover, for all $m>0, l(t)>0$, Assumption 2 holds with

$$
\begin{aligned}
& X=\left[\begin{array}{cc}
1 & 0 \\
-1 & 1
\end{array}\right], \quad U(l)=\left[\begin{array}{ll}
m l^{2}\left(1-\frac{g}{l}\right) & -3 m l^{2}
\end{array}\right], \quad P=\left[\begin{array}{cc}
-1 & 1 \\
0 & -2
\end{array}\right], \\
& R=\left[\begin{array}{cc}
1 & -1 \\
0 & 1
\end{array}\right], \quad L(l)=\left[\begin{array}{c}
\frac{g}{l}-1 \\
-\frac{3 g}{l}
\end{array}\right], \quad Q(l)=\left[\begin{array}{cc}
-\frac{g}{l} & 0 \\
\frac{g}{l} & -\frac{2 g}{l}
\end{array}\right] .
\end{aligned}
$$

Assumption 4 holds with $f(l)=\frac{1}{m l^{2}}$. Furthermore, it is easy to check that Assumptions 1, 5, and 6 do hold.

In a realistic situation, the plant is subjected to disturbances from the environment. If we define $w_{d}$ to be disturbance injected into the plant, and $w_{n}$ to be measurement noise, then the revised model of the plant is

$$
\begin{aligned}
& \dot{x}(t)=A(\alpha(t)) x(t)+B(\alpha(t)) u(t)+w_{d}(t), \quad x(0)=x_{0}, \\
& y(t)=C(\alpha(t)) x(t)+w_{n}(t) .
\end{aligned}
$$

We represent the plant model (14) by the triple $(A(\alpha), B(\alpha), C(\alpha))$. From this point on we fix the plant matrices $A(\alpha), B(\alpha)$, and $C(\alpha)$ as functions of $\alpha$. We will construct a sampled-data controller, which means that $\alpha(t)$ cannot move arbitrarily fast. However we can allow an occasional jump, thus, we fix choices of $T_{0}$, and $\delta_{\alpha}$. The goal of this paper is to develop, under Assumptions 1 to 6, a controller that stabilizes $(A(\alpha), B(\alpha), C(\alpha))$ for every $x_{0} \in \mathbb{R}^{n}, w_{n} \in P C_{\infty}, w_{d} \in P C_{\infty}$, and $\alpha \in P S^{1}\left(\mathcal{A}, T_{0}, \delta_{\alpha}\right)$ when only the plant output $y$ is measurable.

\section{The Controller}

Here, we present the proposed adaptive controller and prove three key lemmas which are essential to the proof of the main result.

\subsection{The Approach}

We seek to stabilize the plant (14) when $\alpha$ is not measurable. We propose a nonlinear periodic controller to achieve this goal. It operates at a period, $T$, and consists of several components:

- a continuous-time filter which is used to provide an upper bound on $\|x(t)\|$ and to scale a probing signal; 


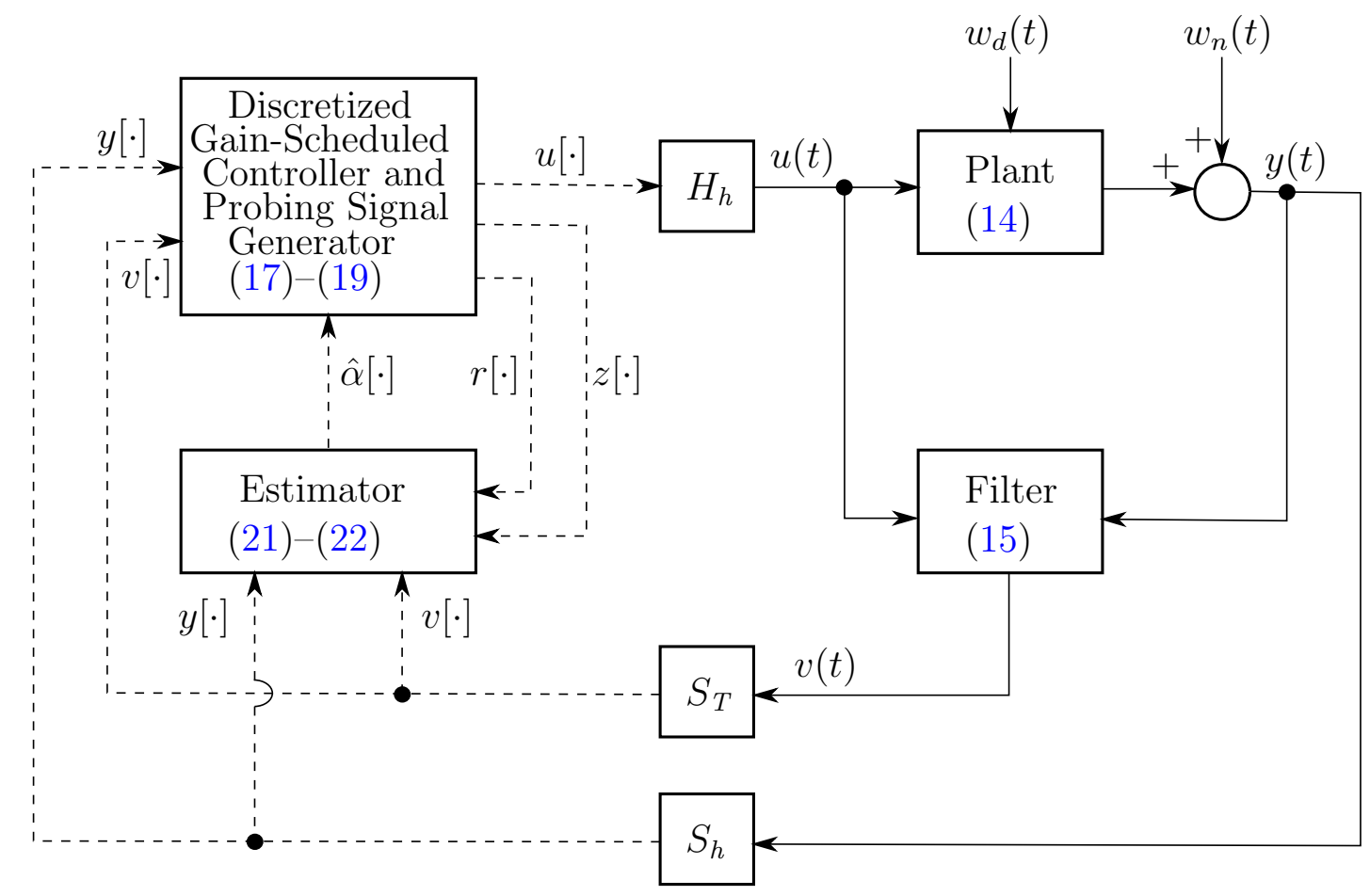

Figure 1: Closed-loop system block diagram ${ }^{4}$.

- a discretized version of the gain-scheduled output feedback controller (11)-(12) with states $z(t)$ and $r(t)$ and $\alpha$ replaced by an estimate $\hat{\alpha}$;

- a sampled-data parameter estimator of $\alpha(t)$ which produces an estimate $\hat{\alpha}[k]$ for use on the control interval $[k T,(k+1) T)$.

The base sampling period is $h=\frac{T}{2}$. A block diagram of the closed-loop system is depicted in Figure 1.

\subsection{The Filter}

By Proposition 1, for each $Q(\alpha) \in \mathcal{H}_{\infty}$ there exists a constant $\lambda<0$ so that, for sufficiently small $T$,

$$
\|I+T Q(\alpha)\|_{\infty} \leq 1+\lambda T, \alpha \in \mathcal{A} .
$$

A method for computing $\lambda$ is given in the proof of [40, Proposition 1]: letting $q_{i j}(\alpha)$ denote the $(i, j)$ th element of $Q(\alpha)$, define

$$
\lambda^{*}:=-\min _{i}\left|q_{i i}(\alpha)+\sum_{j=1, j \neq i}^{m}\right| q_{i j}(\alpha)||,
$$

\footnotetext{
${ }^{4} H_{h}$ is a zero-order-hold operating at period $h$, while $S_{h}$ and $S_{T}$ are ideal samplers operating at periods $h$ and $T$, respectively.
} 
and then fix $\lambda \in\left(\lambda^{*}, 0\right) .^{5}$ The proposed filter is

$$
\dot{v}(t)=\lambda v(t)+\|u(t)\|+\|y(t)\|, \quad v(0)=0 .
$$

It turns out that $v(t)$ provides an upper bound on the size of the state.

Lemma 1. Consider the filter (15) driven by the input and output of the plant (14). There exists a constant $c>0$ so that for every $u \in P C_{\infty}, w_{n} \in P C_{\infty}, w_{d} \in P C_{\infty}, \alpha \in P S^{1}\left(\mathcal{A}, T_{0}, \delta_{\alpha}\right)$, and $x_{0} \in \mathbb{R}^{n}$, the plant state satisfies

$$
\|x(t)\| \leq c \mathrm{e}^{\lambda t}\|x(0)\|+c v(t)+c\left\|w_{n}\right\|_{\infty}+c\left\|w_{d}\right\|_{\infty}, \quad t \geq 0 .
$$

Proof. The proof of Lemma 1 is in the appendix.

\subsection{The Discretized Gain-Scheduled Controller}

With $v(t)$ defined in (15) and with $\hat{\alpha}[k]$ denoting an estimate of $\alpha(k T)$ to be defined shortly, we use a suitably modified discretized version of the LPV controller (11)-(12). The state equation is

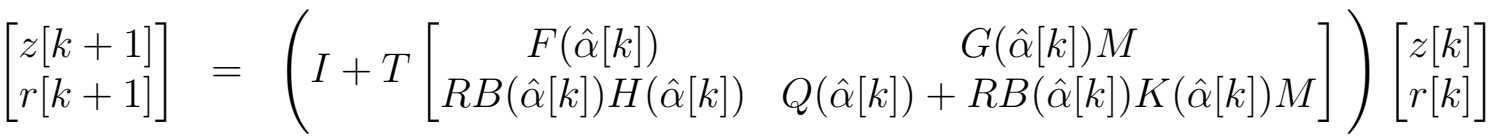

$$
\begin{aligned}
& -T\left[\begin{array}{c}
0 \\
L(\hat{\alpha}[k])
\end{array}\right] y[k], \quad\left[\begin{array}{c}
z(0) \\
r(0)
\end{array}\right]=\left[\begin{array}{l}
0 \\
0
\end{array}\right] .
\end{aligned}
$$

With $\rho \in(0,-\lambda)$, we define a probing signal of the form

$$
\delta(t):= \begin{cases}\rho(v[k]+\|z[k]\|+\|r[k]\|) & t \in[k T, k T+h) \\ -\rho(v[k]+\|z[k]\|+\|r[k]\|) & t \in[k T+h, k T+2 h),\end{cases}
$$

which we add to the discretized version of the output equation of the LPV controller (11)-(12) passed through a zero-order hold, yielding

$$
u(t)=H(\hat{\alpha}[k]) z[k]+K(\hat{\alpha}[k]) M r[k]+\delta(t), t \in[k T,(k+1) T) .
$$

\subsection{The Parameter Estimator}

To motivate the choice of parameter estimator, we examine the simplest relative degree one system

$$
\begin{aligned}
\dot{x}(t) & =a x(t)+b u(t)+w_{d}(t) \\
y(t) & =c x(t)+w_{n}(t),
\end{aligned}
$$

\footnotetext{
${ }^{5}$ Since $Q(\alpha) \in \mathcal{H}_{\infty}$, it can be shown that $\lambda^{*}<0$.
} 
with $a \neq 0$. In light of Assumption 4, the goal here is to estimate the first Markov parameter, $c b$. With $\delta>0$, if we set

$$
u(t)= \begin{cases}\delta & t \in[0, h) \\ -\delta & t \in[h, 2 h)\end{cases}
$$

then

$$
\begin{aligned}
x(h) & =\mathrm{e}^{a h} x(0)+\frac{b \delta}{a}\left(\mathrm{e}^{a h}-1\right)+\int_{0}^{h} \mathrm{e}^{a(h-\tau)} w_{d}(\tau) d \tau, \\
x(2 h) & =\mathrm{e}^{a h} x(h)-\frac{b \delta}{a}\left(\mathrm{e}^{a h}-1\right)+\int_{h}^{2 h} \mathrm{e}^{a(2 h-\tau)} w_{d}(\tau) d \tau \\
& =\mathrm{e}^{2 a h} x(0)+\frac{b \delta}{a}\left(1-2 \mathrm{e}^{a h}+\mathrm{e}^{2 a h}\right)+\int_{0}^{2 h} \mathrm{e}^{a(2 h-\tau)} w_{d}(\tau) d \tau .
\end{aligned}
$$

So it follows that

$$
\begin{aligned}
y(2 h)-2 y(h)+y(0)= & c\left(1-2 \mathrm{e}^{a h}+\mathrm{e}^{2 a h}\right) x(0)+\frac{c b \delta}{a}\left(3-4 \mathrm{e}^{a h}+\mathrm{e}^{2 a h}\right) \\
& +c \int_{0}^{2 h} \mathrm{e}^{a(2 h-\tau)} w_{d}(\tau) d \tau \\
& -2 c \int_{0}^{h} \mathrm{e}^{a(h-\tau)} d \tau+\left[w_{n}(2 h)-2 w_{n}(h)+w_{n}(0)\right]
\end{aligned}
$$

Hence,

$$
\begin{aligned}
\left\|c b-\frac{1}{2 h \delta}(-y(2 h)+2 y(h)-y(0))\right\|= & \mathcal{O}(h)\left(\left\|\frac{x(0)}{\delta}\right\|+1\right) \\
& +\frac{1}{\delta} \mathcal{O}(1)\left\|w_{d}\right\|_{\infty}+\frac{1}{\delta} \mathcal{O}\left(h^{-1}\right)\left\|w_{n}\right\|_{\infty} .
\end{aligned}
$$

So if the last three terms of the RHS of (20) are small, then the LHS provides a good estimate of $c b$. This simple discussion motivates the choice of the estimate in our case where the plant is higher order, the parameter $\alpha$ is time-varying, and the control signal is more complicated. More specifically, with the probing signal defined above in (18), we define the estimate of the first Markov parameter by

$$
\widehat{C B}[k+1]:= \begin{cases}\frac{-y(k T+2 h)+2 y(k T+h)-y(k T)}{2 h \delta[k]} & \text { if } \delta[k] \neq 0 \\ C(\underline{\alpha}) B(\underline{\alpha}) & \text { if } \delta[k]=0,\end{cases}
$$

where $\underline{\alpha}:=\min \{a: a \in \mathcal{A}\}$, which is well-defined because $\mathcal{A}$ is compact by Assumption 3. We then use the estimate of the Markov parameter, under Assumption 4, to form the estimate of $\alpha((k+1) T)$, which we label $\hat{\alpha}[k+1]$ :

$$
\hat{\alpha}[k+1]:=f^{-1}\left(\Pi_{\mathcal{F}}(\widehat{C B}[k+1])\right) .
$$

There are two possible stumbling blocks in the estimation procedure: 
(i) If the probing signal $\delta[k]$ is small relative to the size of $\|x[k]\|$, then the estimation error term of size $\frac{\|x[k]\|}{|\delta[k]|}($ see $(20))$ will be large and the estimate may be inaccurate.

(ii) If the probing signal $\delta[k]$ is small relative to $\left\|w_{d}\right\|_{\infty}$ or to $T^{-1}\left\|w_{n}\right\|_{\infty}$, then the estimate may also be inaccurate.

In the following result we provide sufficient conditions to avoid these problems.

Lemma 2. For every $\epsilon>0$ and $\bar{\delta}>0$, there exist constants $c>0$ and $T_{1}>0$ so that, for every $T \in\left(0, T_{1}\right), k \in \mathbb{Z}_{+}, w_{n} \in P C_{\infty}, w_{d} \in P C_{\infty}, \alpha \in P S^{1}\left(\mathcal{A}, T_{0}, \delta_{\alpha}\right)$, and $x_{0} \in \mathbb{R}^{n}$, when the controller given by (15), (17)-(19), and (21)-(22) is applied to the plant (14), if

(i) $v[k]+\|z[k]\|+\|r[k]\|>\epsilon\|x[k]\|$,

(ii) $v[k]+\|z[k]\|+\|r[k]\|>c\left(T^{-1}\left\|w_{n}\right\|_{\infty}+\left\|w_{d}\right\|_{\infty}\right)$, and

(iii) $\alpha(t)$ is absolutely continuous for $t \in[k T,(k+1) T]$,

then

$$
\|\alpha((k+1) T)-\hat{\alpha}[k+1]\| \leq \bar{\delta}
$$

Proof. The proof of Lemma 2 is in the appendix.

Hence if the probing signal is large relative to the plant state and the noise, and if $\alpha(t)$ is absolutely continuous on $[k T,(k+1) T]$, then the estimate $\hat{\alpha}[k+1]$ of $\alpha[k+1]$ will be accurate, in which case one would expect that the discretized LPV controller (17)-(19) should perform well over the interval $[(k+1) T,(k+2) T)$ (observe that the effect of the probing signal approximately cancels out over an interval). On the other hand, if any of these conditions fail, then the estimate $\hat{\alpha}[k+1]$ may be inaccurate, so the proposed controller may yield an inappropriate control signal on the subsequent interval; however, condition (iii) fails infrequently, while condition (ii) fails only if the controller state is small relative to the size of the noise, so this case should turn out to be unimportant. The tricky condition (i) will be the problematic one, but it will be carefully handled in the proof of the main result.

Before we get to this, however, we first prove that if the estimate $\hat{\alpha}[k+1]$ is accurate, then the closed-loop system behaves well on the following interval $[(k+1) T,(k+2) T)$. To facilitate analysis, we transform the plant state $x$ and controller states $z$ and $r$ using the approach adopted in the proof of [38, Theorem 1]: define $z(t):=z[k]$ and $r(t):=r[k]$ for $t \in[k T,(k+1) T)$, and subsequently define the transformed states

$$
\left[\begin{array}{c}
\bar{x}(t) \\
\bar{z}(t)
\end{array}\right]:=\left[\begin{array}{l}
X \\
Z
\end{array}\right]^{-1}\left[\begin{array}{l}
x(t) \\
z(t)
\end{array}\right], \quad s(t):=R x(t)-r(t) .
$$

At this point we depart from a standard analysis by choosing a norm in just the right way to prove that the closed-loop state is contractive. To this end, it turns out that the system matrix in $\left(\left[\begin{array}{l}\bar{x} \\ \bar{z}\end{array}\right], s\right)$-coordinates is upper block triangular with the $(1,1)$ block being in $\mathcal{H}_{1}$ and the $(2,2)$ 
block being in $\mathcal{H}_{\infty}$. Hence, in light of Proposition 2, it is natural to use a 1-norm on $\left[\begin{array}{l}\bar{x} \\ \bar{z}\end{array}\right]$ and an $\infty$-norm on $s$. Now we are ready to state a property of the closed-loop behaviour over a period.

Lemma 3. There exist constants $T_{2}>0, \hat{\lambda}<0, \bar{\delta}>0$, and $c>0$ together with an invertible matrix $N \in \mathbb{R}^{3 \times 3}$ so that with

$$
p(t):=N\left[\begin{array}{c}
v(t) \\
\left\|\left[\begin{array}{c}
\bar{x}(t) \\
\bar{z}(t)
\end{array}\right]\right\|_{1} \\
\|s(t)\|_{\infty}
\end{array}\right]
$$

for every $T \in\left(0, T_{2}\right), k \in \mathbb{Z}_{+}, w_{n} \in P C_{\infty}, w_{d} \in P C_{\infty}, \alpha \in P S^{1}\left(\mathcal{A}, T_{0}, \delta_{\alpha}\right)$, and $x_{0} \in \mathbb{R}^{n}$, when the controller given by (15), (17)-(19), and (21)-(22) is applied to the plant (14), with $p[k]:=p(k T)$ we have:

(i) In all cases,

$$
\|p(t)-p[k]\|_{\infty} \leq c T\|p[k]\|_{\infty}+c T\left\|w_{n}\right\|_{\infty}+c T\left\|w_{d}\right\|_{\infty}, \quad t \in[k T,(k+1) T)
$$

(ii) In all cases,

$$
\|p[k+1]\|_{\infty} \leq(1+c T)\|p[k]\|_{\infty}+c T\left\|w_{n}\right\|_{\infty}+c T\left\|w_{d}\right\|_{\infty} .
$$

(iii) If $\|\hat{\alpha}[k]-\alpha(k T)\| \leq \bar{\delta}$ and if $\alpha(t)$ is absolutely continuous for $t \in[k T,(k+1) T)$, then

$$
\|p[k+1]\|_{\infty} \leq \mathrm{e}^{\hat{\lambda} T}\|p[k]\|_{\infty}+c T\left\|w_{n}\right\|_{\infty}+c T\left\|w_{d}\right\|_{\infty}
$$

Proof. The proof of Lemma 3 is in the appendix.

\section{The Main Result}

In Lemma 3 we prove that the closed-loop system is well behaved on intervals for which the estimate of $\alpha(t)$ is accurate. We now leverage Lemmas 1-3 to prove that we obtain desirable closed-loop behaviour for all $t \geq 0$. In the following, recall that the initial conditions on the controller states $v(t), z(t)$, and $r(t)$ are zero.

Theorem 2. There exists contants $T_{3}>0, \bar{\lambda}<0$, and $c>0$ so that for every $T \in\left(0, T_{3}\right)$, $w_{n} \in P C_{\infty}, w_{d} \in P C_{\infty}, \alpha \in P S^{1}\left(\mathcal{A}, T_{0}, \delta_{\alpha}\right)$, and $x_{0} \in \mathbb{R}^{n}$, when the controller given by $(15)$, (17)(19), and (21)-(22) is applied to the plant (14), then

$$
\left\|\left[\begin{array}{c}
v(t) \\
x(t) \\
z(t) \\
r(t)
\end{array}\right]\right\| \leq c \mathrm{e}^{\bar{\lambda} t}\left\|\left[\begin{array}{c}
0 \\
x(0) \\
0 \\
0
\end{array}\right]\right\|+c T^{-1}\left\|w_{n}\right\|_{\infty}+c\left\|w_{d}\right\|_{\infty}, \quad t \geq 0 .
$$




\section{Proof.}

\section{Step 1: Bad Estimation Region}

Let $w_{n} \in P C_{\infty}, w_{d} \in P C_{\infty}, \alpha \in P S^{1}\left(\mathcal{A}, T_{0}, \delta_{\alpha}\right)$, and $x_{0} \in \mathbb{R}^{n}$ be arbitrary. Let $c_{1}>0$ be the constant asserted to exist by Lemma 1. Now fix $\epsilon \in\left(0, \frac{1}{2 c_{1}}\right)$. Let $T_{2}>0, \hat{\lambda}<0, \bar{\delta}>0$, and $c_{3}>0$ be the constants, and $N \in \mathbb{R}^{3 \times 3}$ be the invertible matrix, asserted to exist by Lemma 3 ; define $p(t)$ accordingly. Using these choices of $\bar{\delta}$ and $\epsilon$, we let $c_{2}>0$ and $T_{1}>0$ be the constants asserted to exist by Lemma 2 .

Let $T \in\left(0, \max \left\{T_{1}, T_{2}\right\}\right)$ be arbitrary. To proceed, we define two sets of intervals. The first represents times for which the probing signal is too small to ensure a good estimate of $\alpha(t)$, while the second represents times for which the probing signal is of adequate size but is overwhelmed by the noise:

$$
\begin{aligned}
B_{1}(T):=\{t \geq 0: & v(t)+\|z(t)\|+\|r(t)\| \leq \epsilon\|x(t)\|\} \\
B_{2}(T):=\{t \geq 0: & v(t)+\|z(t)\|+\|r(t)\|>\epsilon\|x(t)\| \\
& \left.v(t)+\|z(t)\|+\|r(t)\| \leq c_{2}\left(T^{-1}\left\|w_{n}\right\|_{\infty}+\left\|w_{d}\right\|_{\infty}\right)\right\},
\end{aligned}
$$

and

$$
B(T):=B_{1}(T) \cup B_{2}(T) ;
$$

notice that $B(T)$ is not empty, since $0 \in B_{1}(T)$. Although we expect that the estimate of $\alpha(t)$ will be poor on $B(T)$, quite surprisingly, we are still able to obtain a desirable bound on the state. This is because either:

(i) the controller states are small compared to the plant state, so the filter provides an exponential bound $\left(t \in B_{1}(T)\right)$, or

(ii) the noise is large compared to the closed-loop state $\left(t \in B_{2}(T)\right)$, so it provides a bound on the state.

Claim 1. There exists a constant $c_{4}>0$, so that

$$
\|p(t)\| \leq c_{4} \mathrm{e}^{\lambda t}\|p(0)\|+c_{4} T^{-1}\left\|w_{n}\right\|_{\infty}+c_{4}\left\|w_{d}\right\|_{\infty}, \quad t \in B(T) .
$$

Proof of Claim 1.

First we consider the case of $t \in B_{1}(T)$. By Lemma 1 it follows that

$$
\begin{aligned}
\|x(t)\| & \leq c_{1} \mathrm{e}^{\lambda t}\|x(0)\|+c_{1} v(t)+c_{1}\left\|w_{n}\right\|_{\infty}+c_{1}\left\|w_{d}\right\|_{\infty} \\
& \leq c_{1} \mathrm{e}^{\lambda t}\|x(0)\|+c_{1} \epsilon\|x(t)\|+c_{1}\left\|w_{n}\right\|_{\infty}+c_{1}\left\|w_{d}\right\|_{\infty} ;
\end{aligned}
$$

since $c_{1} \epsilon \in\left(0, \frac{1}{2}\right)$, this yields

$$
\begin{aligned}
\|x(t)\| & \leq \frac{c_{1}}{1-c_{1} \epsilon} \mathrm{e}^{\lambda t}\|x(0)\|+\frac{c_{1}}{1-c_{1} \epsilon}\left\|w_{n}\right\|_{\infty}+\frac{c_{1}}{1-c_{1} \epsilon}\left\|w_{d}\right\|_{\infty} \\
& \leq 2 c_{1} \mathrm{e}^{\lambda t}\|x(0)\|+2 c_{1}\left\|w_{n}\right\|_{\infty}+2 c_{1}\left\|w_{d}\right\|_{\infty} .
\end{aligned}
$$


Additionally, for $t \in B_{1}(T)$, we have that

$$
\begin{aligned}
v(t)+\|z(t)\|+\|r(t)\| & \leq \epsilon\|x(t)\| \leq 2 c_{1} \epsilon \mathrm{e}^{\lambda t}\|x(0)\|+2 c_{1} \epsilon\left\|w_{n}\right\|_{\infty}+2 c_{1} \epsilon\left\|w_{d}\right\|_{\infty} \\
& \leq \mathrm{e}^{\lambda t}\|x(0)\|+\left\|w_{n}\right\|_{\infty}+\left\|w_{d}\right\|_{\infty} .
\end{aligned}
$$

So there exists a constant $c_{5}>0$ so that

$$
\|p(t)\| \leq c_{5} \mathrm{e}^{\lambda t}\|p(0)\|+c_{5}\left\|w_{n}\right\|_{\infty}+c_{5}\left\|w_{d}\right\|_{\infty}, \quad t \in B_{1}(T) .
$$

Now we consider the case of $t \in B_{2}(T)$. By definition of $B_{2}(T)$ it is clear that

$$
\epsilon\|x(t)\|<v(t)+\|z(t)\|+\|r(t)\| \leq c_{2} T^{-1}\left\|w_{n}\right\|_{\infty}+c_{2}\left\|w_{d}\right\|_{\infty},
$$

so there exists a constant $c_{6}>0$ such that

$$
\begin{aligned}
\|p(t)\| & \leq c_{6} T^{-1}\left\|w_{n}\right\|_{\infty}+c_{6}\left\|w_{d}\right\|_{\infty} \\
& \leq c_{6} \mathrm{e}^{\lambda t}\|p(0)\|+c_{6} T^{-1}\left\|w_{n}\right\|_{\infty}+c_{6}\left\|w_{d}\right\|_{\infty}, \quad t \in B_{2}(T) .
\end{aligned}
$$

If we combine (30) and (31), and define $c_{4}:=\max \left\{c_{5}, c_{6}, c_{5} T_{2}\right\}$, then the result follows.

\section{Step 2: Good Estimation Region}

Now define the remaining set of time as

$$
\begin{aligned}
G(T):=[0, \infty) \backslash B(T) \\
= \begin{cases}t \geq 0: & v(t)+\|z(t)\|+\|r(t)\|>\epsilon\|x(t)\|, \\
& \left.v(t)+\|z(t)\|+\|r(t)\|>c_{2}\left(T^{-1}\left\|w_{n}\right\|_{\infty}+\left\|w_{d}\right\|_{\infty}\right)\right\} ;\end{cases}
\end{aligned}
$$

on this set, we expect, roughly speaking, that the estimate of $\alpha(t)$ will be accurate so long as $\alpha(t)$ is smooth.

If $G(T)$ is empty, then Claim 1 provides the desired bound. Now suppose that $G(T)$ is nonempty; then we can write it as a disjoint union of open intervals, possibly an infinite number of them; we will write them as $\left(t_{1}, t_{2}\right),\left(t_{3}, t_{4}\right), \ldots$, with $\left\{t_{i}\right\}$ strictly increasing, which we express concisely as $\left\{\left(t_{i}, t_{i+1}\right): i \in \mathcal{S}\right\}$ with $\mathcal{S} \subseteq\{n \in \mathbb{N}: n$ odd $\}$. The set $G(T)$ is trickier to handle than $B(T)$, with potential issues being the initial partial periods and intervals containing parameter jumps.

The parameter estimator requires a full period in order to return an accurate estimate of $\alpha(t)$. Furthermore, each interval $\left(t_{i}, t_{i+1}\right), i \in \mathcal{S}$, always has a non-empty intersection with intervals of the form $[k T,(k+1) T]$ at the beginning and possibly at the end. This leads us to remove an interval from each end of $\left(t_{i}, t_{i+1}\right)$ and define an associated discrete-time index as follows: with $i \in \mathcal{S}$ odd, define

$$
k_{i}(T):=\operatorname{int}\left(\frac{t_{i}}{T}\right)+2
$$

and

$$
k_{i+1}(T):=\operatorname{int}\left(\frac{t_{i+1}}{T}\right) .
$$

First we obtain a bound on the initial part of the interval $\left(t_{i}, t_{i+1}\right)$, namely $\left[t_{i}, k_{i}(T) T\right]$. 
Claim 2. There exist constants $T_{4} \in\left(0, \min \left\{T_{1}, T_{2}\right\}\right)$ and $c_{7}>0$ such that, for all $T \in\left(0, T_{4}\right)$ and $i \in \mathcal{S}$ :

$$
\|p(t)\| \leq c_{7}\left\|p\left(t_{i}\right)\right\|+c_{7} T\left\|w_{n}\right\|_{\infty}+c_{7} T\left\|w_{d}\right\|_{\infty}, \quad t \in\left[t_{i}, k_{i}(T) T\right] .
$$

Proof of Claim 2.

Let $T \in\left(0, \min \left\{T_{1}, T_{2}\right\}\right)$. From Lemma 3(i)

$$
\begin{aligned}
\left\|p(t)-p\left[k_{i}(T)-2\right]\right\| \leq & c_{3} T\left\|p\left[k_{i}(T)-2\right]\right\|+c_{3} T\left(\left\|w_{n}\right\|_{\infty}+\left\|w_{d}\right\|_{\infty}\right), \\
& t \in\left[\left(k_{i}(T)-2\right) T,\left(k_{i}(T)-1\right) T\right], \\
\left\|p(t)-p\left[k_{i}(T)-1\right]\right\| \leq & c_{3} T\left\|p\left[k_{i}(T)-1\right]\right\|+c_{3} T\left(\left\|w_{n}\right\|_{\infty}+\left\|w_{d}\right\|_{\infty}\right), \\
& t \in\left[\left(k_{i}(T)-1\right) T, k_{i}(T) T\right] .
\end{aligned}
$$

We know that $t_{i} \in\left(\left(k_{i}(T)-2\right) T,\left(k_{i}(T)-1\right) T\right)$; we'd like to use (33) to obtain a bound on $p(t)$ in terms of $p\left(t_{i}\right)$. If we evaluate the LHS of (33) at $t=t_{i}$ and rearrange, we see that

$$
\left(1-c_{3} T\right)\left\|p\left[k_{i}(T)-2\right]\right\| \leq\left\|p\left(t_{i}\right)\right\|+c_{3} T\left(\left\|w_{n}\right\|_{\infty}+\left\|w_{d}\right\|_{\infty}\right),
$$

so if we define $T_{4}:=\min \left\{T_{1}, T_{2}, \frac{1}{2 c_{3}}\right\}$, we see that, for all $T \in\left(0, T_{4}\right)$,

$$
\left\|p\left[k_{i}(T)-2\right]\right\| \leq 2\left\|p\left(t_{i}\right)\right\|+2 c_{3} T\left\|w_{n}\right\|_{\infty}+2 c_{3} T\left\|w_{d}\right\|_{\infty} .
$$

If we now combine this with (33) and (34), then the result follows easily.

Now we need a bound on $\|p(t)\|$ for $t \in\left[k_{i}(T) T, t_{i+1}\right)$. If this interval is empty, then $t_{i+1}-t_{i} \leq$ $2 T$ so we can combine Claims 1 and 2 to yield

$$
\begin{aligned}
\|p(t)\| & \leq c_{7} c_{4} \mathrm{e}^{\lambda t_{i}}\|p(0)\|+\left(c_{7} c_{4} T^{-1}+c_{7} T\right)\left\|w_{n}\right\|_{\infty}+\left(c_{7} c_{4}+c_{7} T\right)\left\|w_{d}\right\|_{\infty} \\
& \leq c_{7} c_{4} \mathrm{e}^{-2 \lambda T} \mathrm{e}^{\lambda t}\|p(0)\|+\left(c_{7} c_{4} T^{-1}+c_{7} T\right)\left\|w_{n}\right\|_{\infty}+\left(c_{7} c_{4}+c_{7} T\right)\left\|w_{d}\right\|_{\infty}, t \in\left[t_{i}, t_{i+1}\right],
\end{aligned}
$$

so there exists a constant $c_{8}>0$ such that

$$
\|p(t)\| \leq c_{8} \mathrm{e}^{\lambda t}\|p(0)\|+c_{8} T^{-1}\left\|w_{n}\right\|_{\infty}+c_{8}\left\|w_{d}\right\|_{\infty}, t \in\left[t_{i}, t_{i+1}\right] .
$$

Now suppose $\left[k_{i}(T), t_{i+1}\right)$ is non-empty; this means that $k_{i+1}(T) \geq k_{i}(T)$. For every $k \in$ $\left[k_{i}(T), k_{i+1}(T)+1\right]$ we have

$$
v[k-1]+\|z[k-1]\|+\|r[k-1]\|>\epsilon\|x[k-1]\|,
$$

and

$$
v[k-1]+\|z[k-1]\|+\|r[k-1]\|>c_{2}\left(T^{-1}\left\|w_{n}\right\|_{\infty}+\left\|w_{d}\right\|_{\infty}\right),
$$

so by Lemma 2 and Lemma 3:

a) if $\alpha(t)$ is absolutely continuous for $t \in[(k-1) T,(k+1) T)$, then $|\alpha(k T)-\hat{\alpha}[k]| \leq \bar{\delta}$, so

$$
\|p[k+1]\| \leq \mathrm{e}^{\hat{\lambda} T}\|p[k]\|+c_{3} T\left\|w_{n}\right\|_{\infty}+c_{3} T\left\|w_{d}\right\|_{\infty} ;
$$


b) if $\alpha(t)$ is not absolutely continuous for $t \in[(k-1) T,(k+1) T)$, then

$$
\|p[k+1]\| \leq\left(1+c_{3} T\right)\|p[k]\|+c_{3} T\left\|w_{n}\right\|_{\infty}+c_{3} T\left\|w_{d}\right\|_{\infty} .
$$

We can now bound the closed-loop state for $t \in\left[k_{i}(T) T,\left(k_{i+1}(T)+1\right) T\right)$; notice that $\left(k_{i+1}(T)+\right.$ 1) $T \geq t_{i+1}$, with equality if and only if $t_{i+1}=\infty$.

Claim 3. For every $\bar{\lambda} \in(\hat{\lambda}, 0)$, there exist constants $T_{3} \in\left(0, T_{4}\right)$ and $c_{9}>0$ so that for all $T \in\left(0, T_{3}\right)$ and $i \in \mathcal{S}$, the following holds:

$$
\|p(t)\| \leq c_{9} \mathrm{e}^{\bar{\lambda}\left(t-k_{i}(T) T\right)}\left\|p\left[k_{i}(T)\right]\right\|+c_{9}\left\|w_{n}\right\|_{\infty}+c_{9}\left\|w_{d}\right\|_{\infty}, \quad t \in\left[k_{i}(T) T,\left(k_{i+1}(T)+1\right) T\right) .
$$

\section{Proof of Claim 3.}

Fix $\bar{\lambda} \in(\hat{\lambda}, 0)$ and let $T \in\left(0, T_{4}\right), i \in \mathcal{S}$, and $k \in\left[k_{i}(T), k_{i+1}(T)\right]$ be arbitrary. From $(35)$ and (36) we see that

$$
\|p[k+1]\| \leq \begin{cases}\mathrm{e}^{\hat{\lambda} T}\|p[k]\|+c_{3} T\left\|w_{n}\right\|_{\infty}+c_{3} T\left\|w_{d}\right\|_{\infty} & \text { if } \alpha(t) \text { is a.c. on }[(k-1) T,(k+1) T), \\ \left(1+c_{3} T\right)\|p[k]\|+c_{3} T\left\|w_{n}\right\|_{\infty}+c_{3} T\left\|w_{d}\right\|_{\infty} & \text { otherwise. }\end{cases}
$$

This gives rise to a time-varying gain

$$
a[k]= \begin{cases}\mathrm{e}^{\hat{\lambda} T} & \text { if } \alpha(t) \text { is a.c. on }[(k-1) T,(k+1) T), \\ \left(1+c_{3} T\right) & \text { otherwise }\end{cases}
$$

with the corresponding state-transition function labelled $\Phi$. Discontinuities in $\alpha(t)$ are spaced by at least $T_{0}$ seconds, so in the time interval $\left[k_{i}(T) T, k T\right]$ there can be at most $\left\lceil\frac{\left(k-k_{i}(T)\right) T}{T_{0}}\right\rceil$ parameter jumps; this means there are at most $2\left\lceil\frac{\left(k-k_{i}(T)\right) T}{T_{0}}\right\rceil$ values of $k$ for which $a[k] \neq \mathrm{e}^{\hat{\lambda} T}$. Because $\Phi\left(k, k_{i}(T)\right)=\prod_{m=k_{i}(T)}^{k-1} a[m]$, it follows that

$$
\begin{aligned}
\left\|\Phi\left(k, k_{i}(T)\right)\right\| & \leq\left(1+c_{3} T\right)^{2\left\lceil\frac{\left(k-k_{i}(T)\right) T}{T_{0}}\right\rceil} \mathrm{e}^{\hat{\lambda} T\left(k-k_{i}(T)-2\left\lceil\frac{\left(k-k_{i}(T)\right) T}{T_{0}}\right\rceil\right)} \\
& =\left[\left(1+c_{3} T\right)^{2} \mathrm{e}^{-2 \hat{\lambda} T}\right]^{\left\lceil\frac{\left(k-k_{i}(T)\right) T}{T_{0}}\right\rceil} \mathrm{e}^{\hat{\lambda} T\left(k-k_{i}(T)\right)}, \quad k=k_{i}(T), \ldots, k_{i+1}(T)+1 .
\end{aligned}
$$

But $\left\lceil\frac{\left(k-k_{i}(T)\right) T}{T_{0}}\right\rceil<\frac{\left(k-k_{i}(T)\right) T}{T_{0}}+1$, so

$$
\left\|\Phi\left(k, k_{i}(T)\right)\right\| \leq\left[\left(1+c_{3} T\right)^{2} \mathrm{e}^{-2 \hat{\lambda} T}\right]^{\frac{\left(k-k_{i}(T)\right) T}{T_{0}}+1} \mathrm{e}^{\hat{\lambda} T\left(k-k_{i}(T)\right)}, \quad k=k_{i}(T), \ldots, k_{i+1}(T)+1 .
$$

We'd like to simplify the first term on the RHS. We claim that there exists a constant $c_{10}>0$ such that

$$
\left\|\Phi\left(k, k_{i}(T)\right)\right\| \leq c_{10} \mathrm{e}^{\bar{\lambda} T\left(k-k_{i}(T)\right)}, \quad k=k_{i}(T), \ldots, k_{i+1}(T)+1 ;
$$

this will be the case if $c_{10}$ satisfies

$$
\left[\left(1+c_{3} T\right)^{2} \mathrm{e}^{-2 \hat{\lambda} T}\right]^{\frac{\left(k-k_{i}(T)\right) T}{T_{0}}+1} \mathrm{e}^{\hat{\lambda} T\left(k-k_{i}(T)\right)} \leq c_{10} \mathrm{e}^{\bar{\lambda} T\left(k-k_{i}(T)\right)}, \quad k=k_{i}(T), \ldots, k_{i+1}(T)+1
$$


which will hold if

$$
c_{10} \geq\left[\left(1+c_{3} T\right)^{2} \mathrm{e}^{-2 \hat{\lambda} T}\right] \mathrm{e}^{\left(\hat{\lambda}-\bar{\lambda}+\frac{1}{T_{0}} \ln \left(\left(1+c_{3} T\right)^{2} \mathrm{e}^{-2 \hat{\lambda} T}\right)\right)\left(k-k_{i}(T)\right) T}, \quad k=k_{i}(T), \ldots, k_{i+1}(T)+1 .
$$

We have $\hat{\lambda}-\bar{\lambda}<0$, so it is clear that we can choose $T_{3} \in\left(0, \min \left\{T_{4}, 1\right\}\right)$ sufficiently small so that

$$
\hat{\lambda}-\bar{\lambda}+\frac{1}{T_{0}} \ln \left(\left(1+c_{3} T\right)^{2} \mathrm{e}^{-2 \hat{\lambda} T}\right) \leq 0, \quad T \in\left(0, T_{3}\right),
$$

and then set $c_{10}=\left(1+c_{3} T_{3}\right)^{2} \mathrm{e}^{-2 \hat{\lambda} T_{3}}$.

Using the upper bound on the size of $\Phi$ given in (41), we can now analyze the difference inequality (38) and obtain an upper bound on the size of $p$ :

$$
\begin{aligned}
\|p[k]\| & \leq\left\|\Phi\left(k, k_{i}(T)\right)\right\| \times\left\|p\left[k_{i}(T)\right]\right\|+\left(\sum_{m=k_{i}(T)}^{k-1}\|\Phi(k-1, m)\|\right)\left(c_{3} T\left\|w_{n}\right\|_{\infty}+c_{3} T\left\|w_{d}\right\|_{\infty}\right) \\
& \leq c_{10} \mathrm{e}^{\bar{\lambda} T\left(k-k_{i}(T)\right)}\left\|p\left[k_{i}(T)\right]\right\|+c_{10}\left(\sum_{m=0}^{k-\left(k_{i}(T)+1\right)} \mathrm{e}^{\bar{\lambda} T m}\right)\left(c_{3} T\left\|w_{n}\right\|_{\infty}+c_{3} T\left\|w_{d}\right\|_{\infty}\right),
\end{aligned}
$$

for $k=k_{i}(T), \ldots, k_{i+1}(T)+1$. We can obtain an upper bound on the summation with

$$
\sum_{m=0}^{k-\left(k_{i}(T)+1\right)} \mathrm{e}^{\bar{\lambda} T m} \leq \sum_{m=0}^{\infty}\left(\mathrm{e}^{\bar{\lambda} T}\right)^{m}=\frac{1}{1-\mathrm{e}^{\bar{\lambda} T}}=\mathcal{O}\left(T^{-1}\right)
$$

so there exists a constant $c_{11}>0$ such that for all $T \in\left(0, T_{3}\right)$ :

$$
\begin{aligned}
\|p[k]\| \leq & c_{11} \mathrm{e}^{\bar{\lambda} T\left(k-k_{i}(T)\right)}\left\|p\left[k_{i}(T)\right]\right\|+c_{11}\left\|w_{n}\right\|_{\infty}+c_{11}\left\|w_{d}\right\|_{\infty}, \\
& k=k_{i}(T), \ldots, k_{i+1}(T)+1 .
\end{aligned}
$$

Finally, by Lemma 3(i), we have that

$$
\|p(t)\| \leq\left(1+c_{3} T\right) c_{11} \mathrm{e}^{\bar{\lambda} T\left(k-k_{i}(T)\right)}\left\|p\left[k_{i}(T)\right]\right\|+\left(\left(1+c_{3} T\right) c_{11}+c_{3} T\right)\left(\left\|w_{n}\right\|_{\infty}+\left\|w_{d}\right\|_{\infty}\right)
$$

for $t \in\left[k_{i}(T) T,\left(k_{i+1}(T)+1\right) T\right)$, so there exists a constant $c_{9}>0$ such that for all $T \in\left(0, T_{3}\right)$ :

$$
\|p(t)\| \leq c_{9} \mathrm{e}^{\bar{\lambda}\left(t-k_{i}(T) T\right)}\left\|p\left[k_{i}(T)\right]\right\|+c_{9}\left\|w_{n}\right\|_{\infty}+c_{9}\left\|w_{d}\right\|_{\infty}, \quad t \in\left[k_{i}(T) T, t_{i+1}\right) .
$$

Now restrict $T \in\left(0, T_{3}\right)$. We can bound all of $t \in\left(t_{i}, t_{i+1}\right), i \in \mathcal{S}$ odd, as follows. By Claim 1 ,

$$
\left\|p\left(t_{i}\right)\right\| \leq c_{4} \mathrm{e}^{\lambda t_{i}}\|p(0)\|+c_{4} T^{-1}\left\|w_{n}\right\|_{\infty}+c_{4}\left\|w_{d}\right\|_{\infty} .
$$

So by Claim 2

$$
\begin{aligned}
\|p(t)\| \leq & c_{7}\left\|p\left(t_{i}\right)\right\|+c_{7} T\left\|w_{n}\right\|_{\infty}+c_{7} T\left\|w_{d}\right\|_{\infty} \\
\leq & c_{7} c_{4} \mathrm{e}^{-2 \lambda T} \mathrm{e}^{\lambda t}\|p(0)\|+\left(c_{7} c_{4} T^{-1}+c_{7} T\right)\left\|w_{n}\right\|_{\infty} \\
& +\left(c_{7} c_{4}+c_{7} T\right)\left\|w_{d}\right\|_{\infty}, \quad t \in\left[t_{i}, k_{i}(T) T\right] ;
\end{aligned}
$$


if we set $c_{12}:=\max \left\{c_{4} c_{7} \mathrm{e}^{-2 \lambda T_{3}}, c_{4} c_{7}+c_{7} T_{3}^{2}, c_{4} c_{7}+c_{7} T_{3}\right\}$, then

$$
\|p(t)\| \leq c_{12} \mathrm{e}^{\lambda t}\|p(0)\|+c_{12} T^{-1}\left\|w_{n}\right\|_{\infty}+c_{12}\left\|w_{d}\right\|_{\infty}, \quad t \in\left[t_{i}, k_{i}(T) T\right] .
$$

By Claim 3, we have

$$
\begin{aligned}
\|p(t)\| & \leq c_{9} \mathrm{e}^{\bar{\lambda}\left(t-k_{i}(T) T\right)}\left\|p\left[k_{i}(T)\right]\right\|+c_{9}\left\|w_{n}\right\|_{\infty}+c_{9}\left\|w_{d}\right\|_{\infty} \\
& \leq c_{9} c_{12} \mathrm{e}^{\bar{\lambda} t}\|p(0)\|+\left(c_{9} c_{12} T^{-1}+c_{9}\right)\left\|w_{n}\right\|_{\infty}+\left(c_{9} c_{12}+c_{9}\right)\left\|w_{d}\right\|_{\infty}, \quad t \in\left[k_{i}(T) T, t_{i+1}\right] .
\end{aligned}
$$

Then, with $c_{13}:=\max \left\{c_{9} c_{12}+c_{9}, \quad c_{12}, \quad c_{9} c_{12}+c_{9} T_{3}\right\}$, we end up with

$$
\|p(t)\| \leq c_{13} \mathrm{e}^{\bar{\lambda} t}\|p(0)\|+c_{13} T^{-1}\left\|w_{n}\right\|_{\infty}+c_{13}\left\|w_{d}\right\|_{\infty}, \quad t \in\left(t_{i}, t_{i+1}\right) .
$$

\section{Step 3: Final Bound}

By Claim 1, for all $t \in B(T)$ we have

$$
\|p(t)\| \leq c_{4} \mathrm{e}^{\lambda t}\|p(0)\|+c_{4} T^{-1}\left\|w_{n}\right\|_{\infty}+c_{4}\left\|w_{d}\right\|_{\infty},
$$

and using (43), for all $t \in G(T)$ we have

$$
\|p(t)\| \leq c_{13} \mathrm{e}^{\bar{\lambda} t}\|p(0)\|+c_{13} T^{-1}\left\|w_{n}\right\|_{\infty}+c_{13}\left\|w_{d}\right\|_{\infty} .
$$

Defining $c:=\max \left\{c_{4}, c_{13}\right\}$ gives the desired result.

\section{A Simulation Example}

We present a simulation example of our controller design for a plant that cannot be stabilized by an LTI controller. Consider the system

$$
\begin{aligned}
& \dot{x}(t)=\left[\begin{array}{cc}
\alpha(t) & 0 \\
0 & -1
\end{array}\right] x(t)+\left[\begin{array}{c}
1 \\
\alpha(t)
\end{array}\right] u(t), \\
& y(t)=\left[\begin{array}{ll}
1 & 1
\end{array}\right] x(t) .
\end{aligned}
$$

The time-varying parameter $\alpha(t)$ takes values in the set $\mathcal{A}=[-9.5,-1.5] \cup[1.5,9.5]$. For our purposes, we assume that the derivative of $\alpha$ is bounded above by $\delta_{\alpha}=100$ and that there is a minimum time between jumps of $T_{0}=0.5$ seconds. If we freeze $\alpha$, the plant transfer function is

$$
\frac{(1+\alpha)(s+1-\alpha)}{(s-\alpha)(s+1)},
$$

which has a zero at $s=\alpha-1>0$ and a pole at $s=\alpha$ for all $\alpha \in[1.5,9.5]$. Indeed, the frozen plant will have both an unstable pole and a non-minimum phase zero when $\alpha \in[1.5,9.5]$, with the zero being slower than the pole (a particularly nasty setup). The first Markov parameter is $f(\alpha)=C(\alpha) B(\alpha)=1+\alpha$. It is routine to check that this plant satisfies Assumptions 1, 4 and 5 . 
This plant is difficult to control; it cannot be stabilized by an LTI controller for all $\alpha \in \mathcal{A}$. This fact follows from the study of strong stabilization in [41, Corollary 12], e.g., you cannot simultaneously stabilize the plant corresponding to $\alpha=2$ with that corresponding to $\alpha=-2$ using a single LTI controller.

Following Theorem 1, the matrices

$$
X=\left[\begin{array}{cc}
0.1 & 0 \\
0 & 1
\end{array}\right], \quad U(\alpha)=\left[-1.5|\alpha|\left(\frac{1}{10+\alpha}+\frac{1}{10-\alpha}\right) \quad 0\right], \quad R=\left[\begin{array}{cc}
0.1 & 0 \\
0 & 1
\end{array}\right], \quad L(\alpha)=\left[\begin{array}{c}
-\frac{10}{33}|\alpha| \\
0
\end{array}\right],
$$

satisfy (8), (9). These matrices are used to get the controller described in Section 4.3. Finally, the filter pole is chosen to be $\lambda=-1$ by the method described in Section 4.2.

The discretized gain-scheduled controller (17) is given by

$r[k+1]=\left[\begin{array}{cc}1+T\left(\hat{\alpha}[k]-\frac{10}{33}|\hat{\alpha}[k]|-15|\hat{\alpha}[k]|\left(\frac{1}{10+\hat{\alpha}[k]}+\frac{1}{10-\hat{\alpha}[k]}\right)\right) & -\frac{10}{33} T|\hat{\alpha}[k]| \\ -150 T|\hat{\alpha}[k]| \hat{\alpha}[k]\left(\frac{1}{10+\hat{\alpha}[k]}+\frac{1}{10-\hat{\alpha}[k]}\right) & 1+T\end{array}\right] r[k]+\left[\begin{array}{c}\frac{10}{33} T|\hat{\alpha}[k]| \\ 0\end{array}\right] y[k]$,

and the filter (15) is given by

$$
\dot{v}(t)=-v(t)+|u(t)|+|y(t)| .
$$

To simulate the closed-loop system, we choose $h=0.001$ seconds (yielding $T=0.002$ seconds) and $\rho=0.25$.

Remark 2. The criterion for selecting the parameters $\lambda$ and $\rho$ was discussed in Sections 4.2 and 4.3, respectively. Briefly, to select $\lambda$, we first compute the class $\mathcal{H}_{\infty}$ matrix $Q(\alpha)$ satisfying (9) and then use it to compute $\lambda^{*}<0$ which characterizes the allowable range of $\lambda \in\left(\lambda^{*}, 0\right)$. After fixing a choice of $\lambda$, we are free to pick any $\rho \in(0,-\lambda)$. The selection of a sufficiently small sampling period $h$ is best addressed through simulation.

The parameter $\alpha(t)$ switches between the trajectories

$$
\alpha(t)=5.5+4 \sin (25 t), \quad \alpha(t)=-5.5-4 \sin (25 t),
$$

and spends 1 second following the former trajectory, and 0.5 seconds following the latter, between jumps. Uniformly distributed noise with $\left\|w_{n}\right\|_{\infty}=0.05$ and $\left\|w_{d}\right\|_{\infty}=0.05$ is injected in the system for $t \in[5,15)$; otherwise, the system is noise-free. The plant initial condition is set to $x(0)=\left[\begin{array}{c}10 \\ 1\end{array}\right]$.

The simulation results are presented in Figures 2 and 3. We see that the output of the plant is bounded in response to the initial condition and the measurement noise. Furthermore, we see from Figure 3 that the parameter estimator works quite well (on average), even in the presence of noise. While the control signal is fairly large, and the plant state becomes quite large as well, this is mostly due to the nastiness of the plant and the difficulty of the problem considered in this paper. Indeed, in Figure 4 we compare our adaptive controller to that of the original LPV controller; we see that, even in a best case scenario where the parameter $\alpha$ is known, the size of the state vector can become large. That being said, the sizeable difference starting at $t=5$ seconds stems from the larger effect of the noise in the adaptive setting than in the LPV setting; this is the price that we pay for having to estimate the plant parameter $\alpha$. Nevertheless, the proposed adaptive controller guarantees a stable closed-loop system even in the presence of frequent parameter jumps. 

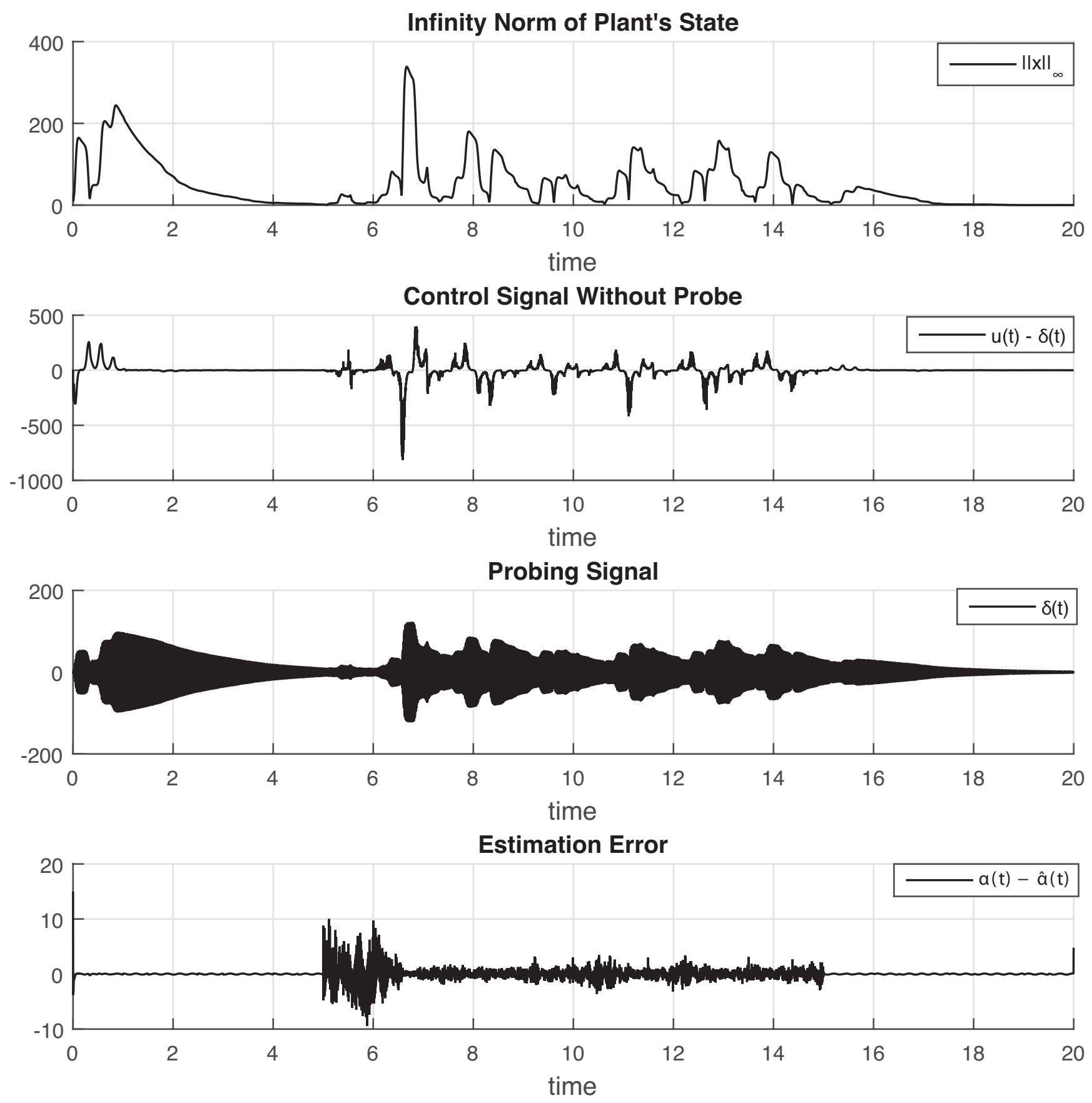

Figure 2: The norm of the plant state, control signal, probing signal, and estimation error (the noise turns on at 5 seconds and off at 15 seconds).

\section{Summary and Conclusions}

In this paper we consider the problem of designing a controller to adaptively stabilize an uncertain linear parameter-varying (LPV) plant. This plant can be rapidly time-varying and can have unstable zero dynamics (the time-varying counterpart of being non-minimum phase). While there are a number of results in the literature for the situation of an uncertain plant with stable zero dynamics, the case of unstable zero dynamics, however, is very challenging. Indeed, results related 


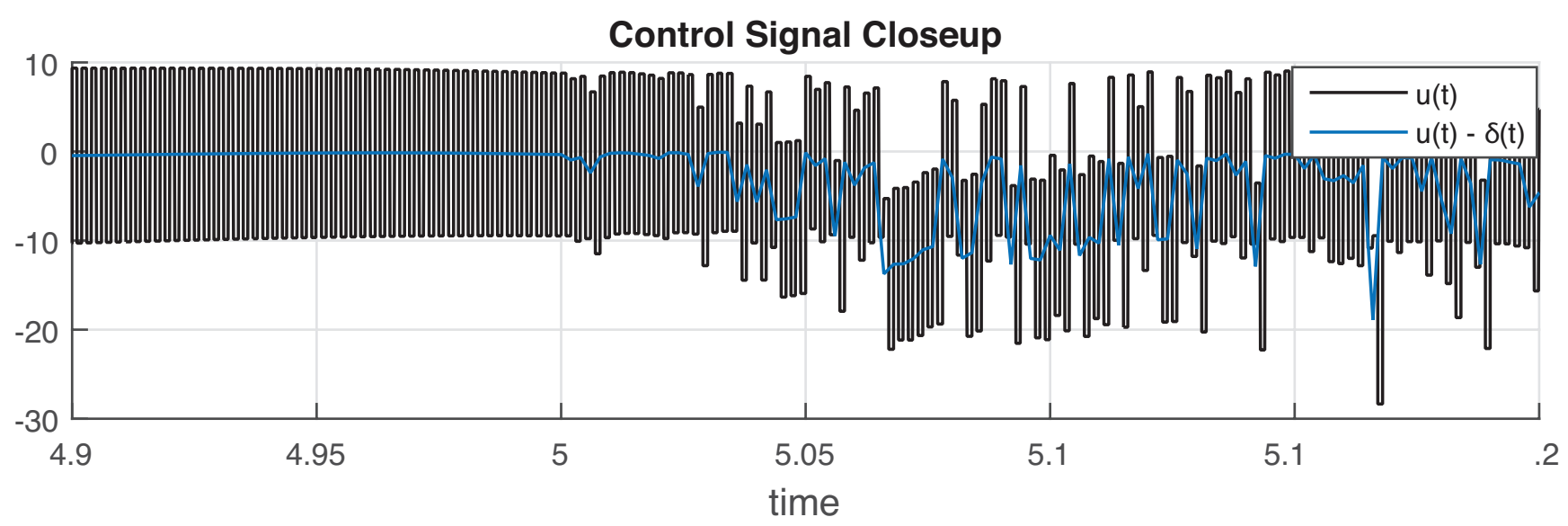

Time-Varying Parameter vs. Estimate Closeup

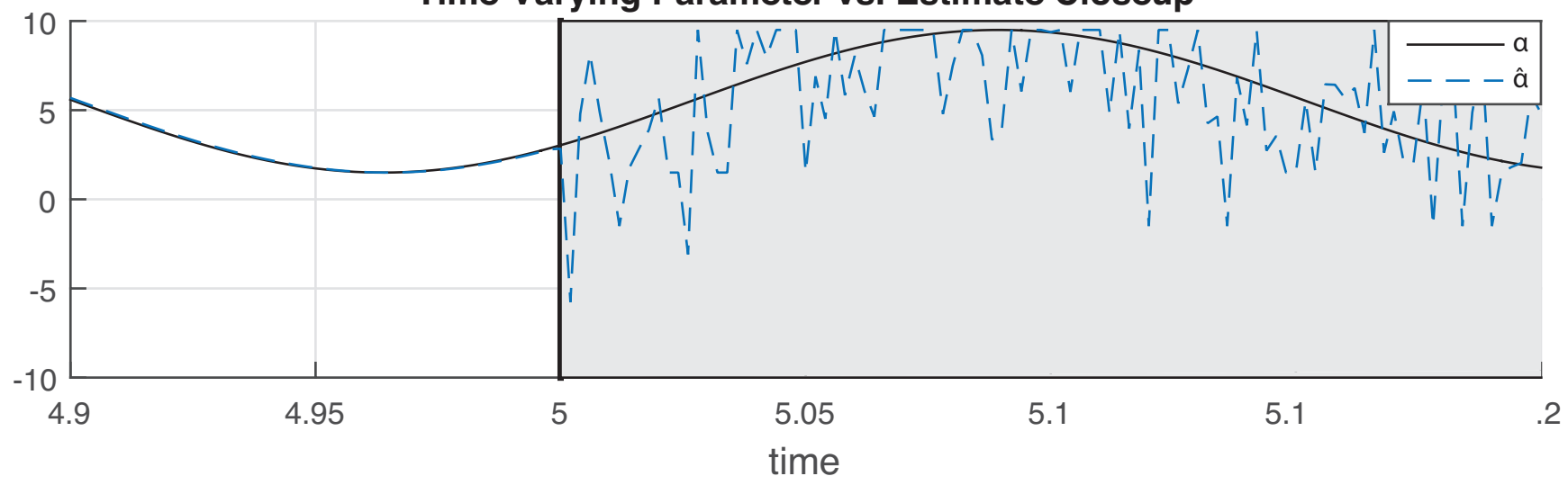

Figure 3: Close up of the control signal and parameter estimate/parameter value (the noise turns on at 5 seconds and off at 15 seconds).

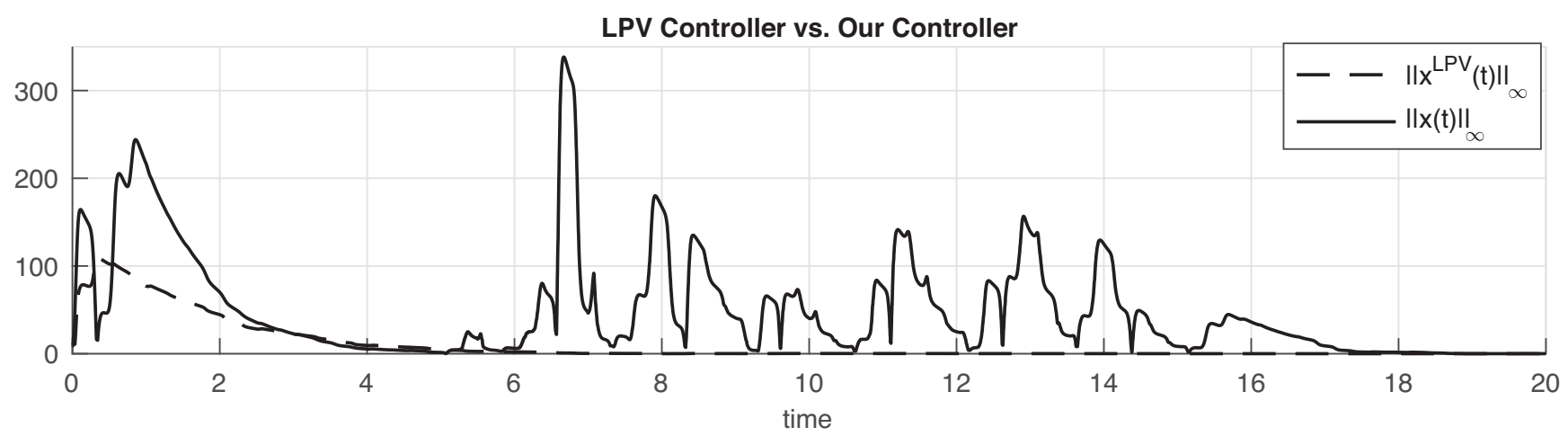

Figure 4: The $\infty$-norm of the plant's state vector versus time when: (i) our proposed controller is used (solid line) and, (ii) the parameter $\alpha$ is known and the LPV controller from [38] is used (dashed line).

to plants having unstable zero dynamics suffer very stringent conditions on the plant.

In this paper a new approach is provided based on results in gain scheduling, particularly [38]. A controller design is presented based on a discretized version of a gain-scheduled output feedback controller, with the scheduling variable replaced by an estimate. The estimate is generated by 
a discrete-time estimator which uses the state of a filter to appropriately scale a probing signal. Under suitable assumptions on the LPV plant, it is proven that if the controller sampling period is small enough, then the closed-loop system is exponentially stable with bounded gain on the noise in the presence of rapid time-variation and persistent parameter jumps. Furthermore, the controller can tolerate noisy measurements and disturbances injected into the state, although the noise gain may be large. Finally, an illustrative example of a plant with unstable zero dynamics is provided.

At the moment, the plant is limited to being single-input single-output and having a scalarvalued scheduling variable; however, we would like to extend this approach to multiple-input multiple-output plants with vector-valued scheduling variables. Furthermore, due to the difficult nature of handling a plant with a time-varying state-to-output relationship, a major structural assumption that the plant be relative degree one for each value of the scheduling parameter is required. We would like to weaken this assumption so that the applicability of the approach can be extended.

\section{Appendix A Proofs}

\section{A.1 Proof of Lemma 1}

Let $u \in P C_{\infty}, w_{n} \in P C_{\infty}, w_{d} \in P C_{\infty}, \alpha \in P S^{1}\left(\mathcal{A}, T_{0}, \delta_{\alpha}\right)$, and $x_{0} \in \mathbb{R}^{n}$ be arbitrary. The proof is organized into three steps:

1. Motivated by equation (9), define matrices $R_{e}, A_{e}(\alpha)$, and $C_{e}(\alpha)$, with $R_{e}$ non-singular, so that $R_{e} A_{e}(\alpha)+L(\alpha) C_{e}(\alpha)=Q(\alpha) R_{e}$.

2. Use the equation from step 1 to bound a yet to be defined extended state $x_{e}(t)$.

3. Use the bound on $x_{e}(t)$ to bound the plant's state $x(t)$.

\section{Step 1}

Let $v$ be the number of columns of the constant full rank matrix $R$ from Assumption 2. Let $\bar{R} \in \mathbb{R}^{v \times(v-n)}$ be any matrix so that $R_{e}:=\left[\begin{array}{ll}R & \bar{R}\end{array}\right]$ is non-singular, and then define

$$
\left[\begin{array}{l}
A_{12}(\alpha) \\
A_{22}(\alpha)
\end{array}\right]:=R_{e}^{-1} Q(\alpha) \bar{R}
$$

where $A_{12}(\alpha) \in \mathbb{R}^{n \times(v-n)}, A_{22}(\alpha) \in \mathbb{R}^{(v-n) \times(v-n)}$. Then, by the definition of $R, R_{e}, A_{12}$, and $A_{22}$,

$$
\underbrace{\left[\begin{array}{cc}
A(\alpha) & A_{12}(\alpha) \\
0 & A_{22}(\alpha)
\end{array}\right]}_{=: A_{e}(\alpha)}+R_{e}^{-1} L(\alpha) \underbrace{\left[\begin{array}{cc}
C(\alpha) & 0
\end{array}\right]}_{=: C_{e}(\alpha)}=R_{e}^{-1} Q(\alpha) R_{e} .
$$

\section{Step 2}

Consider the control system

$$
\begin{aligned}
{\left[\begin{array}{l}
\dot{x}_{1}(t) \\
\dot{x}_{2}(t)
\end{array}\right] } & =\left[\begin{array}{cc}
A(\alpha(t)) & A_{12}(\alpha(t)) \\
0 & A_{22}(\alpha(t))
\end{array}\right]\left[\begin{array}{l}
x_{1}(t) \\
x_{2}(t)
\end{array}\right]+\left[\begin{array}{c}
B(\alpha(t)) \\
0
\end{array}\right] u(t)+\left[\begin{array}{c}
w_{d}(t) \\
0
\end{array}\right] \\
y_{e}(t) & =\left[\begin{array}{ll}
C(\alpha(t)) & 0
\end{array}\right]\left[\begin{array}{l}
x_{1}(t) \\
x_{2}(t)
\end{array}\right]+w_{n}(t),
\end{aligned}
$$


where $x_{1}(t) \in \mathbb{R}^{n}, x_{2}(t) \in \mathbb{R}^{v-n}$. Define $x_{e}:=\left(x_{1}, x_{2}\right)$; then

$$
\begin{aligned}
& \dot{x}_{e}(t)=\left(\left[\begin{array}{cc}
A(\alpha(t)) & A_{12}(\alpha(t)) \\
0 & A_{22}(\alpha(t))
\end{array}\right]+R_{e}^{-1} L(\alpha(t))\left[\begin{array}{cc}
C(\alpha(t)) & 0
\end{array}\right]\right) x_{e}(t) \\
& -R_{e}^{-1} L(\alpha(t))\left(y_{e}(t)-w_{n}(t)\right)+\left[\begin{array}{c}
B(\alpha(t)) \\
0
\end{array}\right] u(t)+\left[\begin{array}{c}
w_{d}(t) \\
0
\end{array}\right] .
\end{aligned}
$$

Using (45), we can write

$$
\begin{aligned}
\dot{x}_{e}(t)= & R_{e}^{-1} Q(\alpha(t)) R_{e} x_{e}(t)-R_{e}^{-1} L(\alpha(t)) y_{e}(t)+R_{e}^{-1} L(\alpha(t)) w_{n}(t) \\
& +\left[\begin{array}{c}
B(\alpha(t)) \\
0
\end{array}\right] u(t)+\left[\begin{array}{c}
w_{d}(t) \\
0
\end{array}\right] .
\end{aligned}
$$

The solution to this differential equation, for $t \geq 0$ and any $x_{e}(0) \in \mathbb{R}^{v}$, is

$$
\begin{aligned}
x_{e}(t)= & \Phi(t, 0) x_{e}(0)+\int_{0}^{t} \Phi(t, \tau)\left(\left[\begin{array}{c}
B(\alpha(\tau)) \\
0
\end{array}\right] u(\tau)-R_{e}^{-1} L(\alpha(\tau)) y_{e}(\tau)\right) \mathrm{d} \tau \\
& +\int_{0}^{t} \Phi(t, \tau)\left(R_{e}^{-1} L(\alpha(\tau)) w_{n}(\tau)+\left[\begin{array}{c}
w_{d}(\tau) \\
0
\end{array}\right]\right) \mathrm{d} \tau,
\end{aligned}
$$

where $\Phi(t, 0)$ is the state transition function of the unforced system

$$
\dot{z}(t)=R_{e}^{-1} Q(\alpha(t)) R_{e} z(t), \quad t \geq 0 .
$$

Consider the change of coordinates $p=R_{e} z$, where $R_{e}$ is the non-singular matrix from Step 1 . Then $\dot{p}=Q(\alpha(t)) p$ with $Q(\alpha(t)) \in \mathcal{H}_{\infty}$ so that, by Proposition 2 , there exist $\gamma \geq 1$ and $\lambda<0$ such that

$$
\|p(t)\| \leq \gamma \mathrm{e}^{\lambda t}\|p(0)\|, \quad t \geq 0 .
$$

Again, following the proof of [40, Proposition 1], the constant $\lambda$ can be taken to be the same as that in the filter (15). Then there exists a constant $c_{1}$ such that for all $t \geq 0,\|z(t)\| \leq c_{1} \mathrm{e}^{\lambda t}\|z(0)\|$, so we conclude that $\|\Phi(t, 0)\| \leq c_{1} \mathrm{e}^{\lambda t}$ for $t \geq 0$. Using this bound on $\Phi(t, 0)$ in the expression for $x_{e}(t)$ we get

$$
\left\|x_{e}(t)\right\| \leq c \mathrm{e}^{\lambda t}\left\|x_{e}(0)\right\|+c \int_{0}^{t} \mathrm{e}^{\lambda(t-\tau)}\left(\|u(\tau)\|+\left\|y_{e}(\tau)\right\|\right) \mathrm{d} \tau+c\left(\left\|w_{n}\right\|_{\infty}+\left\|w_{d}\right\|_{\infty}\right),
$$

with $c$ defined as

$$
c:=c_{1} \max _{\alpha \in \mathcal{A}}\left\{1,\left\|R_{e}^{-1}\right\|\|L(\alpha)\|,\|B(\alpha)\|,-\frac{\left\|R_{e}^{-1}\right\|\|L(\alpha)\|}{\lambda},-\frac{1}{\lambda}\right\}
$$

\section{Step 3}

The subspace $\left\{x_{e}: x_{2}=0\right\}$ is invariant for the extended system. Let $x(t)$ be the solution of the 
plant's ODE (14a) with initial condition $x(0)$. Then the initial condition $x_{e}(0)=(x(0), 0)$ admits the solution $x_{e}(t)=(x(t), 0)$ and $y_{e}(t)=y(t)$. Therefore, using $(46)$,

$$
\|x(t)\|=\left\|x_{e}(t)\right\| \leq c \mathrm{e}^{\lambda t}\|x(0)\|+c \int_{0}^{t} \mathrm{e}^{\lambda(t-\tau)}(\|u(\tau)\|+\|y(\tau)\|) d \tau+c\left(\left\|w_{n}\right\|_{\infty}+\left\|w_{d}\right\|_{\infty}\right) .
$$

The solution $v(t)$ of (15) equals the integral in the RHS of the above inequality, so we get the desired result.

\section{A.2 Proof of Lemma 2}

In the proofs of Lemma 2 and Lemma 3, we utilize a crude bound on the maximum growth of the plant's state over a single period.

Proposition 3. There exist constants $\bar{T}>0$ and $c>0$ so that for every $T \in(0, \bar{T})$, $w_{d} \in P C_{\infty}$, $\alpha \in P S^{1}\left(\mathcal{A}, T_{0}, \delta_{\alpha}\right)$, and $x[k] \in \mathbb{R}^{n}$, when the controller given by (15), (17)-(19), and (21), (22) is applied to the plant (14):

$$
\|x(t)-x[k]\| \leq c T\left(v[k]+\left\|\left[\begin{array}{c}
\bar{x}[k] \\
\bar{z}[k]
\end{array}\right]\right\|_{1}+\|s[k]\|_{\infty}+\left\|w_{d}\right\|_{\infty}\right), \quad t \in[k T,(k+1) T] .
$$

Proof of Proposition 3.

Let $w_{d} \in P C_{\infty}, \alpha \in P S^{1}\left(\mathcal{A}, T_{0}, \delta_{\alpha}\right)$, and $x[k] \in \mathbb{R}^{n}$ be arbitrary. The solution $x(t)$ to (14) with initial condition $x[k]$ satisfies

$$
x(t)-x[k]=\int_{k T}^{t}\left(A(\alpha(\tau)) x[k]+B(\alpha(\tau)) u(\tau)+w_{d}(\tau)\right) \mathrm{d} \tau+\int_{k T}^{t} A(\alpha(\tau))(x(\tau)-x[k]) \mathrm{d} \tau .
$$

Taking norms and substituting the expression for $u(t)$ over $[k T,(k+1) T]$, we get the upper bound

$$
\begin{aligned}
\|x(t)-x[k]\| \leq & T\left(\max _{\alpha \in \mathcal{A}}\|A(\alpha)\|\|x[k]\|+\max _{\alpha \in \mathcal{A}}\|B(\alpha)\|\left(\max _{\alpha \in \mathcal{A}}\|H(\alpha)\|+\rho\right)\|z[k]\|\right. \\
& \left.+\max _{\alpha \in \mathcal{A}}\|B(\alpha)\|\left(\max _{\alpha \in \mathcal{A}}\|K(\alpha)\|\|M\|+\rho\right)\|r[k]\|+\max _{\alpha \in \mathcal{A}}\|B(\alpha)\| \rho v[k]+\left\|w_{d}\right\|_{\infty}\right) \\
& +\max _{\alpha \in \mathcal{A}}\|A(\alpha)\| \int_{k T}^{t}\|x(t)-x[k]\| \mathrm{d} \tau, \quad t \in[k T,(k+1) T] .
\end{aligned}
$$

Using the definition of $\bar{x}, \bar{z}$, and $s$ given in (24) and invoking the Bellman-Gronwall inequality, there exist constants $c_{1}>0$ and $c_{2}>0$ so that

$$
\|x(t)-x[k]\| \leq c_{1} T\left(v[k]+\left\|\left[\begin{array}{c}
\bar{x}[k]] \\
\bar{z}[k]
\end{array}\right]\right\|_{1}+\|s[k]\|_{\infty}\right) \mathrm{e}^{c_{2} T}+c_{1} T\left\|w_{d}\right\|_{\infty} \mathrm{e}^{c_{2} T} .
$$

Then, for sufficiently small $\bar{T}$, there exists a constant $c>0$ so that, for all $T \in(0, \bar{T})$, 


$$
\|x(t)-x[k]\| \leq c T\left(v[k]+\left\|\left[\begin{array}{c}
\bar{x}[k] \\
\bar{z}[k]
\end{array}\right]\right\|_{1}+\|s[k]\|_{\infty}+\left\|w_{d}\right\|_{\infty}\right) .
$$

Proof of Lemma 2.

Fix $\epsilon>0$ and $\bar{\delta}>0$; let $w_{n} \in P C_{\infty}, w_{d} \in P C_{\infty}, \alpha \in P S^{1}\left(\mathcal{A}, T_{0}, \delta_{\alpha}\right), k \in \mathbb{Z}_{+}$, and $x_{0} \in \mathbb{R}^{n}$ be arbitrary. We start with a claim.

Claim 1. There exist constants $c>0$ and $\bar{T}>0$, so that if $T \in(0, \bar{T}), v[k]+\|z[k]\|+\|r[k]\|>$ $\epsilon\|x[k]\|$, and $\alpha(t)$ is a.c. for $t \in[k T,(k+1) T]$, then

$$
\|C(\alpha((k+1) T)) B(\alpha((k+1) T))-\widehat{C B}[k+1]\| \leq c T+c \frac{\left\|w_{d}\right\|_{\infty}}{\delta[k]}+c T^{-1} \frac{\left\|w_{n}\right\|_{\infty}}{\delta[k]} .
$$

Proof of Claim 1.

By hypothesis, $v[k]+\|z[k]\|+\|r[k]\|>\epsilon\|x[k]\|$, which implies that $\delta[k] \neq 0$. Then

$$
\begin{aligned}
-2 h \widehat{C B}[k+1] \delta[k]= & y(k T+2 h)-2 y(k T+h)+y(k T) \\
= & \left(y_{n f}(k T+2 h)-y_{n f}(k T+h)\right)-\left(y_{n f}(k T+h)-y_{n f}(k T)\right) \\
& +w_{n}(k T+2 h)-2 w_{n}(k T+h)+w_{n}(k T),
\end{aligned}
$$

where, with some abuse of notation, $y_{n f}(t):=C(t) x(t)$ is the noise-free output signal. By this definition of $y_{n f}(t)$, it follows that

$$
\dot{y}_{n f}(t)=(\dot{C}(t)+C(t) A(t)) x(t)+C(t) B(t) u(t)+C(t) w_{d}(t)
$$

for almost every $t \in[k T,(k+1) T]$. Then, by the Fundamental Theorem of Calculus and the structure of the control signal (19), we have

$$
\begin{aligned}
y_{n f}(k T+h)-y_{n f}(k T)= & \int_{k T}^{k T+h}(\dot{C}(\tau)+C(\tau) A(\tau)) x(\tau) \mathrm{d} \tau+\int_{k T}^{k T+h} C(\tau) B(\tau) \mathrm{d} \tau \delta[k] \\
& +\int_{k T}^{k T+h} C(\tau) B(\tau) \mathrm{d} \tau(H(\hat{\alpha}[k]) z[k]+K(\hat{\alpha}[k]) M r[k]) \\
& +\int_{k T}^{k T+h} C(\tau) w_{d}(\tau) \mathrm{d} \tau .
\end{aligned}
$$

In a similar fashion, 


$$
\begin{aligned}
y_{n f}(k T+2 h)-y_{n f}(k T+h)= & \int_{k T+h}^{k T+2 h}(\dot{C}(\tau)+C(\tau) A(\tau)) x(\tau) \mathrm{d} \tau \\
& -\int_{k T+h}^{k T+2 h} C(\tau) B(\tau) \mathrm{d} \tau \delta[k] \\
& +\int_{k T+h}^{k T+2 h} C(\tau) B(\tau) \mathrm{d} \tau(H(\hat{\alpha}[k]) z[k]+K(\hat{\alpha}[k]) M r[k]) \\
& +\int_{k T+h}^{k T+2 h} C(\tau) w_{d}(\tau) \mathrm{d} \tau .
\end{aligned}
$$

Substituting the previous two expressions into (49) yields

$$
\begin{aligned}
-2 h \widehat{C B}[k+1] \delta[k]= & \int_{k T+h}^{k T+2 h}(C(\tau) A(\tau)+\dot{C}(\tau)) x(\tau) \mathrm{d} \tau+\int_{k T+h}^{k T+2 h} C(\tau) w_{d}(\tau) \mathrm{d} \tau \\
& +\int_{k T+h}^{k T+2 h} C(\tau) B(\tau) \mathrm{d} \tau(H(\hat{\alpha}[k]) z[k]+K(\hat{\alpha}[k]) M r[k]) \\
& -\int_{k T}^{k T+h} C(\tau) B(\tau) \mathrm{d} \tau(H(\hat{\alpha}[k]) z[k]+K(\hat{\alpha}[k]) M r[k]) \\
& -\int_{k T}^{k T+2 h} C(\tau) B(\tau) \mathrm{d} \tau \delta[k]-\int_{k T}^{k T+h}(C(\tau) A(\tau)+\dot{C}(\tau)) x(\tau) \mathrm{d} \tau \\
& -\int_{k T}^{k T+h} C(\tau) w_{d}(\tau) \mathrm{d} \tau+w_{n}(k T+2 h)-2 w_{n}(k T+h)+w_{n}(k T) \\
+ & -2 h C(k T+2 h) B(k T+2 h) \delta[k] \\
+ & \int_{k T}^{k T+2 h}(C(k T+2 h) B(k T+2 h)-C(\tau) B(\tau)) \mathrm{d} \tau \delta[k] \\
+ & \int_{k T+h}^{k T+2 h}((C(\tau) A(\tau)+\dot{C}(\tau)) x(\tau)-(C(\tau-h) A(\tau-h)+\dot{C}(\tau-h)) x(\tau-h)) \mathrm{d} \tau \\
+ & +\int_{k T+h}^{k T+2 h}(C(\tau) B(\tau)-C(\tau-h) B(\tau-h)) \mathrm{d} \tau(H(\hat{\alpha}[k]) z[k]+K(\hat{\alpha}[k]) M r[k]) \\
+ & +\int_{k T+h}^{k T+2 h} C(\tau) w_{d}(\tau) \mathrm{d} \tau-\int_{k T}^{k T+h} C(\tau) w_{d}(\tau) \mathrm{d} \tau
\end{aligned}
$$

Then we have

$$
\|C(k T+2 h) B(k T+2 h)-\widehat{C B}[k+1]\| \leq \frac{1}{2 h} \int_{k T}^{k T+2 h}\|C(k T+2 h) B(k T+2 h)-C(\tau) B(\tau)\| \mathrm{d} \tau
$$




$$
\begin{aligned}
& +\frac{1}{2 h \delta[k]} \int_{k T+h}^{k T+2 h}\|(C(\tau) A(\tau)+\dot{C}(\tau)) x(\tau)-(C(\tau-h) A(\tau-h)+\dot{C}(\tau-h)) x(\tau-h)\| \mathrm{d} \tau \\
& +\frac{1}{2 h \delta[k]} \int_{k T+h}^{k T+2 h}\|C(\tau) B(\tau)-C(\tau-h) B(\tau-h)\| \mathrm{d} \tau(H(\hat{\alpha}[k]) z[k]+K(\hat{\alpha}[k]) M r[k]) \\
& +\frac{1}{2 h \delta[k]}\left\|\int_{k T+h}^{k T+2 h} C(\tau) w_{d}(\tau) \mathrm{d} \tau-\int_{k T}^{k T+h} C(\tau) w_{d}(\tau) \mathrm{d} \tau\right\| \\
& +\frac{1}{2 h \delta[k]}\left\|w_{n}(k T+2 h)-2 w_{n}(k T+h)+w_{n}(k T)\right\| .
\end{aligned}
$$

Utilizing order notation, Proposition 3, and applying Assumption 5 to bound ${ }^{6}$ the Lipschitz continuous functions, we can write this concisely as

$$
\begin{aligned}
\|C(k T+2 h) B(k T+2 h)-\widehat{C B}[k+1]\|= & \mathcal{O}(T)+\mathcal{O}(T) \frac{v[k]+\|x[k]\|+\|z[k]\|+\|r[k]\|}{\delta[k]} \\
& +\mathcal{O}(1) \frac{\left\|w_{d}\right\|_{\infty}}{\delta[k]}+\mathcal{O}\left(T^{-1}\right) \frac{\left\|w_{n}\right\|_{\infty}}{\delta[k]} .
\end{aligned}
$$

By hypothesis we have

$$
\frac{\|x[k]\|+v[k]+\|z[k]\|+\|r[k]\|}{\delta[k]} \leq\left(\frac{1}{\epsilon}+1\right) \frac{(v[k]+\|z[k]\|+\|r[k]\|)}{\rho(v[k]+\|z[k]\|+\|r[k]\|)}=\frac{1}{\rho}\left(\frac{1}{\epsilon}+1\right),
$$

so for all $T \in(0, \bar{T})$, with $\bar{T}$ sufficiently small, we get the desired result:

$$
\|C((k+1) T) B((k+1) T)-\widehat{C B}[k+1]\|=\mathcal{O}(T)+\mathcal{O}(1) \frac{\left\|w_{d}\right\|_{\infty}}{\delta[k]}+\mathcal{O}\left(T^{-1}\right) \frac{\left\|w_{n}\right\|_{\infty}}{\delta[k]}
$$

By hypotheses (i) and (iii) and Claim 1, the bound (48) holds. By hypothesis (ii) and the definition of $\delta[k]$,

$$
\frac{\left\|w_{n}\right\|_{\infty}}{T \delta[k]}+\frac{\left\|w_{d}\right\|_{\infty}}{\delta[k]}<\frac{1}{\rho c}
$$

With $c_{1}$ the constant from Claim 1, substituting (50) into (48) yields

$$
\|C((k+1) T) B((k+1) T)-\widehat{C B}[k+1]\|<c_{1}\left(T+\frac{1}{\rho c}\right) .
$$

We want to bound $\| C\left(\alpha((k+1) T) B\left(\alpha((k+1) T)-\Pi_{\mathcal{F}}(\widehat{C B}[k+1]) \|\right.\right.$, so we must account for the effect of the projection, $\Pi_{\mathcal{F}}$. To ensure that $\Pi_{\mathcal{F}}$ projects onto the interval of $\mathcal{F}$ containing $C((k+1) T) B((k+1) T)$, it is sufficient that the upper bound in (51) be less than half the minimum distance between intervals of $\mathcal{F}$. By Assumptions 3 and 5, the image of $\mathcal{A}$ under $f$ has

\footnotetext{
${ }^{6}$ It is important to note that by using Proposition 3 the estimation error will be upper bounded by the entire closed-loop state, including $v(t)$.
} 
the form $\mathcal{F}=\left[\underline{f}_{1}, \bar{f}_{1}\right] \cup \cdots \cup\left[\underline{f}_{q}, \bar{f}_{q}\right]$, where $\bar{f}_{i}<\underline{f}_{i+1}, i=1, \ldots, q-1$, for some $q \in \mathbb{N}$. Let $d_{\min }:=\min _{j}\left(\underline{f}_{j+1}-\bar{f}_{j}\right), j=1, \ldots, q-1$, and let $T_{1}:=\frac{1}{c_{1}} \min \left\{\frac{d_{\min }}{4}, \frac{\bar{\delta}}{2 \ell}\right\}$, where $\ell$ is the Lipschitz constant of $f^{-1}$ on $\mathcal{F}$. Then, from $(51)$, for any $c>\frac{c_{1}}{\rho} \max \left\{\frac{4}{d_{\min }}, \frac{2 \ell}{\bar{\delta}}\right\}$ and any $T \in\left(0, T_{1}\right)$, we get

$$
\|C((k+1) T) B((k+1) T)-\widehat{C B}[k+1]\|<\frac{c_{1} d_{\min }}{4 c_{1}}+\frac{c_{1} \rho d_{\min }}{4 c_{1} \rho}=\frac{d_{\min }}{2},
$$

so it follows that

$$
\left\|C((k+1) T) B((k+1) T)-\Pi_{\mathcal{F}}(\widehat{C B}[k+1])\right\| \leq\|C((k+1) T) B((k+1) T)-\widehat{C B}[k+1]\| .
$$

By Assumption 4 we have

$$
\begin{aligned}
\|\alpha((k+1) T)-\hat{\alpha}[k+1]\| & \leq \ell\left\|C((k+1) T) B((k+1) T)-\Pi_{\mathcal{F}}(\widehat{C B}[k+1])\right\| \\
& <c_{1} \ell\left(T+\frac{1}{\rho c}\right)<\frac{c_{1} \ell \bar{\delta}}{2 c_{1} \ell}+\frac{c_{1} \ell \rho \bar{\delta}}{2 c_{1} \ell \rho}=\bar{\delta}
\end{aligned}
$$

where we have used the fact that $\rho c>\frac{2 c_{1} \ell}{\bar{\delta}}$.

\section{A.3 Proof of Lemma 3}

Let $w_{n} \in P C_{\infty}, w_{d} \in P C_{\infty}, \alpha \in P S^{1}\left(\mathcal{A}, T_{0}, \delta_{\alpha}\right), k \in \mathbb{Z}_{+}$, and $x_{0} \in \mathbb{R}^{n}$ be arbitrary.

\section{Proof of (iii)}

In order to accomplish this, we proceed as follows:

- Analyze all states at the sample points (Steps 1, 2, and 3),

- Upper bound a transformed closed-loop state (Step 4).

\section{Step 1 - Filter Sample Point Analysis}

At the sample points, the filter (15) satisfies

$$
v[k+1]=\mathrm{e}^{\lambda T} v[k]+\int_{k T}^{(k+1) T} \mathrm{e}^{\lambda((k+1) T-\tau)}\|u(\tau)\| \mathrm{d} \tau+\int_{k T}^{(k+1) T} \mathrm{e}^{\lambda((k+1) T-\tau)}\|y(\tau)\| \mathrm{d} \tau .
$$

By (19), the input term satisfies

$$
\begin{array}{r}
\int_{k T}^{(k+1) T} \mathrm{e}^{\lambda((k+1) T-\tau)}\|u(\tau)\| \mathrm{d} \tau=-\frac{1}{\lambda}\left(1-\mathrm{e}^{\lambda h}\right)\left[\|H(\hat{\alpha}[k]) z[k]+K(\hat{\alpha}[k]) M r[k]+\delta[k]\| \mathrm{e}^{\lambda h}\right. \\
+\|H(\hat{\alpha}[k]) z[k]+K(\hat{\alpha}[k]) M r[k]-\delta[k]\|]
\end{array}
$$




$$
\leq-\frac{1}{\lambda}\left(-\frac{\lambda T}{2}\right)\left(2\left(\max _{\alpha \in \mathcal{A}}\|H(\alpha)\|+\rho\right)\|z[k]\|+2\left(\max _{\alpha \in \mathcal{A}}\|K(\alpha)\|\|M\|+\rho\right)\|r[k]\|+2 \rho v[k]\right) .
$$

Employing order notation, we can write this compactly as

$$
\int_{k T}^{(k+1) T} \mathrm{e}^{\lambda((k+1) T-\tau)}\|u(\tau)\| \mathrm{d} \tau=T \rho v[k]+\mathcal{O}(T)\left\|\left[\begin{array}{c}
\bar{x}[k] \\
\bar{z}[k]
\end{array}\right]\right\|_{1}+\mathcal{O}(T)\|s[k]\|_{\infty},
$$

where $\bar{x}, \bar{z}$, and $s$ are defined in (24). The output term satisfies

$$
\begin{aligned}
\int_{k T}^{(k+1) T} \mathrm{e}^{\lambda((k+1) T-\tau)}\|y(\tau)\| \mathrm{d} \tau \leq & \int_{k T}^{(k+1) T} \mathrm{e}^{\lambda((k+1) T-\tau)} \mathrm{d} \tau \overbrace{\|C(\alpha(k T)) x[k]\|}^{=\mathcal{O}(1)\|x[k]\|} \\
& +\int_{k T}^{(k+1) T} \mathrm{e}^{\lambda((k+1) T-\tau)} \underbrace{(\underbrace{\|C(\alpha(\tau))-C(\alpha(k T))\|\|x[k]\|}_{\text {bound using Proposition } 3}}_{=\mathcal{O}(T)\|x[k]\|} \\
& +\underbrace{\|C(\alpha(\tau))\|\|x(\tau)-x[k]\|}+\left\|w_{n}\right\|_{\infty}) \mathrm{d} \tau .
\end{aligned}
$$

Employing order notation, and utilizing Assumption 5 and Proposition 3, we can write this compactly as

$$
\begin{aligned}
\int_{k T}^{(k+1) T} \mathrm{e}^{\lambda((k+1) T-\tau)}\|y(\tau)\| \mathrm{d} \tau= & \mathcal{O}(T)\left\|\left[\begin{array}{l}
\bar{x}[k]] \\
\bar{z}[k]
\end{array}\right]\right\|_{1}+\mathcal{O}\left(T^{2}\right) v[k]+\mathcal{O}\left(T^{2}\right)\|s[k]\|_{\infty} \\
& +\mathcal{O}(T)\left\|w_{n}\right\|_{\infty}+\mathcal{O}\left(T^{2}\right)\left\|w_{d}\right\|_{\infty} .
\end{aligned}
$$

Combining these upper bounds, for sufficiently small $T$ there exist constants $e_{1}>0, \gamma_{1}>0$, $\gamma_{2}>0$, and $w_{1}>0$ such that, for all such $T$ and all $k \in \mathbb{Z}_{+}$,

$$
\begin{aligned}
v[k+1] \leq & \left(1+(\lambda+\rho) T+e_{1} T^{2}\right) v[k]+\gamma_{1} T\left\|\left[\begin{array}{c}
\bar{x}[k] \\
\bar{z}[k]
\end{array}\right]\right\|_{1}+\gamma_{2} T\|s[k]\|_{\infty} \\
& +w_{1} T\left\|w_{n}\right\|_{\infty}+w_{1} T^{2}\left\|w_{d}\right\|_{\infty} .
\end{aligned}
$$

\section{Step 2 - Controller Sample Point Analysis}

Starting with $z[k+1]$, define

$$
e_{z 1}[k]:=T(F(\hat{\alpha}[k])-F(\alpha(k T))) z[k]+T(G(\hat{\alpha}[k])-G(\alpha(k T))) M r[k],
$$

so that we can write

$$
z[k+1]=\left[\begin{array}{lll}
0 & I+T F(\alpha(k T)) & T G(\alpha(k T)) M
\end{array}\right]\left[\begin{array}{c}
x[k] \\
z[k] \\
r[k]
\end{array}\right]+e_{z 1}[k] .
$$

By Assumptions 5 and 6 (see Remark 1 ), there exists a constant $\ell_{1}>0$ so that we have 


$$
\left\|e_{z 1}[k]\right\| \leq T \ell_{1}\|\tilde{\alpha}(k T)\|\left\|\left[\begin{array}{c}
\bar{x}[k]] \\
\bar{z}[k]
\end{array}\right]\right\|_{1}+T \ell_{1}\|\tilde{\alpha}(k T)\|\|s[k]\|_{\infty},
$$

where $\tilde{\alpha}(k T)=\alpha(k T)-\hat{\alpha}[k]$ denotes the parameter estimation error at time $k T$. By hypothesis $\|\tilde{\alpha}(k T)\| \leq \bar{\delta}$, so

$$
\left\|e_{z 1}[k]\right\| \leq T \ell_{1} \bar{\delta}\left\|\left[\begin{array}{c}
\bar{x}[k] \\
\bar{z}[k]
\end{array}\right]\right\|_{1}+T \ell_{1} \bar{\delta}\|s[k]\|_{\infty} .
$$

We can treat $r[k+1]$ similarly and define

$$
\begin{aligned}
e_{z 2}[k]:= & -T(L(\hat{\alpha}[k])-L(\alpha(k T))) C(\alpha(k T)) x[k] \\
& +T R(B(\hat{\alpha}[k]) H(\hat{\alpha}[k])-B(\alpha(k T)) H(\alpha(k T))) z[k] \\
& +T((Q(\hat{\alpha}[k])-Q(\alpha(k T)))+R(B(\hat{\alpha}[k]) K(\hat{\alpha}[k])-B(\alpha(k T)) K(\alpha(k T)))) r[k] \\
& -T L(\hat{\alpha}[k]) w_{n}[k],
\end{aligned}
$$

so that

$$
\begin{aligned}
r[k+1]= & -T L(\alpha(k T)) C(\alpha(k T)) x[k]+T R B(\alpha(k T)) H(\alpha(k T)) z[k] \\
& +(I+T(Q(\alpha(k T))+R B(\alpha(k T)) K(\alpha(k T)) M)) r[k] \\
& +e_{z 2}[k] .
\end{aligned}
$$

Again, by Assumptions 5 and 6, there exists a constant $\ell_{2}>0$ such that

$$
\left\|e_{z 2}[k]\right\| \leq T \ell_{2} \bar{\delta}\left\|\left[\begin{array}{l}
\bar{x}[k] \\
\bar{z}[k]
\end{array}\right]\right\|_{1}+T \ell_{2} \bar{\delta}\|s[k]\|_{\infty}+T \ell_{2}\left\|w_{n}\right\|_{\infty} .
$$

Finally, we will use

$$
e_{u}[k]:=(H(\hat{\alpha}[k])-H(\alpha(k T))) z[k]+(K(\hat{\alpha}[k])-K(\alpha(k T))) M r[k],
$$

to write

$$
\begin{aligned}
& u[k]-\delta(t)=H(\hat{\alpha}[k]) z[k]+K(\hat{\alpha}[k]) M r[k]
\end{aligned}
$$

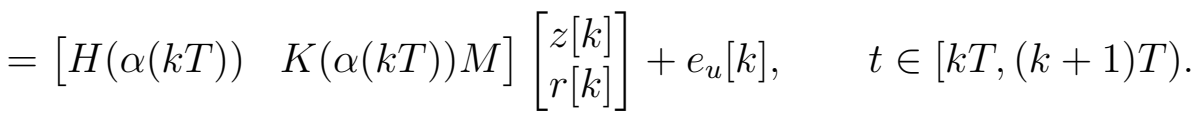

Again, by Assumptions 5 and 6 , there exists a constant $\ell_{3}>0$ such that

$$
\left\|e_{u}[k]\right\| \leq \ell_{3} \bar{\delta}\left\|\left[\begin{array}{l}
\bar{x}[k] \\
\bar{z}[k]
\end{array}\right]\right\|_{1}+\ell_{3} \bar{\delta}\|s[k]\|_{\infty}
$$

\section{Step 3 - Plant Sample Point Analysis}

The value of the plant state at time $t=(k+1) T$ is 


$$
x[k+1]=x[k]+\int_{k T}^{(k+1) T} A(\alpha(\tau)) x(\tau) \mathrm{d} \tau+\int_{k T}^{(k+1) T} B(\alpha(\tau)) u(\tau) \mathrm{d} \tau+\int_{k T}^{(k+1) T} w_{d}(\tau) \mathrm{d} \tau,
$$

so that, using the structure of the control signal (19),

$$
\begin{aligned}
x[k+1]= & (I+T A(\alpha(k T))) x[k]+T B(\alpha(k T))\left([H(\alpha(k T)) \quad K(\alpha(k T))]\left[\begin{array}{c}
z[k] \\
r[k]
\end{array}\right]+e_{u}[k]\right) \\
& +\int_{k T}^{(k+1) T}(A(\alpha(\tau))-A(\alpha(k T))) \mathrm{d} \tau x[k]+\int_{k T}^{(k+1) T} A(\alpha(\tau))(x(\tau)-x[k]) \mathrm{d} \tau \\
& +\int_{k T}^{(k+1) T}(B(\alpha(\tau))-B(\alpha(k T))) \mathrm{d} \tau(H(\hat{\alpha}[k]) z[k]+K(\hat{\alpha}[k]) M r[k]) \\
& +\int_{k T}^{(k+1) T} B(\alpha(\tau)) \delta(\tau) \mathrm{d} \tau+\int_{k T}^{(k+1) T} w_{d}(\tau) \mathrm{d} \tau .
\end{aligned}
$$

Define

$$
\begin{aligned}
e_{p}[k]:= & \int_{k T}^{(k+1) T}(A(\alpha(\tau))-A(\alpha(k T))) \mathrm{d} \tau x[k]+\int_{k T}^{(k+1) T} A(\alpha(\tau))(x(\tau)-x[k]) \mathrm{d} \tau \\
& +\int_{k T}^{(k+1) T}(B(\alpha(\tau))-B(\alpha(k T))) \mathrm{d} \tau(H(\hat{\alpha}[k]) z[k]+K(\hat{\alpha}[k]) M r[k]) \\
& +\int_{k T}^{(k+1) T} B(\alpha(\tau)) \delta(\tau) \mathrm{d} \tau+\int_{k T}^{(k+1) T} w_{d}(\tau) \mathrm{d} \tau,
\end{aligned}
$$

to be able to compactly write

$$
\begin{aligned}
x[k+1]= & {\left[\begin{array}{lll}
I+T A(\alpha(k T)) & T B(\alpha(k T)) H(\alpha(k T)) & T B(\alpha(k T)) K(\alpha(k T)) M]
\end{array}\left[\begin{array}{c}
x[k] \\
z[k] \\
r[k]
\end{array}\right]\right.} \\
& +T B(\alpha(k T)) e_{u}[k]+e_{p}[k] .
\end{aligned}
$$

By Assumptions 5 and 6 (see Remark 1) and Proposition 3, there exists a constant $\ell_{4}>0$ such that for small $T$

$$
\left\|e_{p}[k]\right\| \leq T^{2} \ell_{4} v[k]+T^{2} \ell_{4}\left\|\left[\begin{array}{c}
\bar{x}[k] \\
\bar{z}[k]
\end{array}\right]\right\|_{1}+T^{2} \ell_{4}\|s[k]\|_{\infty}+T \ell_{4}\left\|w_{d}\right\|_{\infty} .
$$

\section{Step 4 - Closed-Loop Difference Inequality}

Combining the analysis of the plant and the controller, we obtain

$$
\begin{aligned}
{\left[\begin{array}{l}
x[k+1] \\
z[k+1] \\
r[k+1]
\end{array}\right]=} & \left(I+T\left[\begin{array}{ccc}
A(\alpha(k T)) & B(\alpha(k T)) H(\alpha(k T)) & B(\alpha(k T)) K(\alpha(k T)) M \\
0 & F(\alpha(k T)) & G(\alpha(k T)) M \\
-L(\alpha(k T)) C(\alpha(k T)) & R B(\alpha(k T)) H(\alpha(k T)) & Q(\alpha(k T))+R B(\alpha(k T)) K(\alpha(k T)) M
\end{array}\right]\right)\left[\begin{array}{l}
x[k] \\
z[k] \\
r[k]
\end{array}\right] \\
& +\left[\begin{array}{c}
T B(\alpha(k T)) e_{u}[k]+e_{p}[k] \\
e_{z 1}[k] \\
e_{z 2}[k]
\end{array}\right] .
\end{aligned}
$$


In $(\bar{x}, \bar{z}, s)$-coordinates, making use of (9) and (13), we have

$$
\begin{aligned}
& {\left[\begin{array}{c}
\bar{x}[k+1] \\
\bar{z}[k+1] \\
s[k+1]
\end{array}\right]=\left(I+T\left[\begin{array}{c}
P(\alpha(k T)) \\
0
\end{array}\right]=\left[\begin{array}{c}
X \\
Z
\end{array}\right]^{-1}\left[\begin{array}{c}
B(\alpha(k T)) K(\alpha(k T)) M \\
G(\alpha(k T)) M \\
Q(\alpha(k T))
\end{array}\right]\right)\left[\begin{array}{c}
\bar{x}[k] \\
\bar{z}[k] \\
s[k]
\end{array}\right]} \\
& +\left[\begin{array}{c}
{\left[\begin{array}{c}
X \\
Z
\end{array}\right]^{-1}\left[\begin{array}{c}
T B(\alpha(k T)) e_{u}[k]+e_{p}[k] \\
e_{z 1}[k]
\end{array}\right]} \\
T R B(\alpha(k T)) e_{u}[k]+R e_{p}[k]-e_{z 2}[k]
\end{array}\right] .
\end{aligned}
$$

To construct the decrescent norm, we define a difference inequality and apply two similarity transformations. We start by upper bounding the transformed plant and controller states:

$$
\begin{aligned}
\|\left[\begin{array}{l}
\bar{x}[k+1]] \\
\bar{z}[k+1]
\end{array} \|_{1} \leq\right. & \|I+T P(\alpha(k T))\|_{1}\left\|\left[\begin{array}{l}
\bar{x}[k]] \\
\bar{z}[k]
\end{array}\right]\right\|_{1} \\
& \left.+T \|\left[\begin{array}{c}
X \\
Z
\end{array}\right] \begin{array}{c}
B(\alpha(k T)) K(\alpha(k T)) M \\
G(\alpha(k T)) M
\end{array}\right]\left\|_{1}\right\| s[k] \|_{1} \\
& +\left\|\left[\begin{array}{c}
X \\
Z
\end{array}\right]^{-1}\left[\begin{array}{c}
T B(\alpha(k T)) e_{u}[k]+e_{p}[k] \\
e_{z 1}[k]
\end{array}\right]\right\|_{1}
\end{aligned}
$$

Next we take advantage of the $\mathcal{H}_{1}$ property that $P(\alpha)$ enjoys: by Proposition 1 there exists a constant $\lambda_{1}<0$ so that $\|I+T P(\alpha)\|_{1} \leq 1+\lambda_{1} T$ for all sufficiently small $T$; similarly, we can take advantage of the $\mathcal{H}_{\infty}$ property that $Q(\alpha)$ enjoys: there exists a constant $\lambda_{2}<0$ so that $\|I+T Q(\alpha)\|_{\infty} \leq 1+\lambda_{2} T$ for all sufficiently small $T$; clearly we can choose $\lambda_{1}$ and $\lambda_{2}$ so that $\lambda_{2}<\lambda_{1}<0$. Then using (53), (55), and (56), there exists constants $e_{2}>0, \gamma_{3}>0$, and $w_{2}>0$ such that for small $T$

$$
\begin{aligned}
\left\|\left[\begin{array}{l}
\bar{x}[k+1] \\
\bar{z}[k+1]
\end{array}\right]\right\|_{1} \leq & \left(1+\lambda_{1} T+e_{2} T(\bar{\delta}+T)\right)\left\|\left[\begin{array}{l}
\bar{x}[k] \\
\bar{z}[k]
\end{array}\right]\right\|_{1}+\left(\gamma_{3} T+e_{2} T(\bar{\delta}+T)\right)\|s[k]\|_{\infty} \\
& +e_{2} T^{2} v[k]+w_{2} T\left\|w_{d}\right\|_{\infty} .
\end{aligned}
$$

In a similar fashion, we have an upper bound on $s[k+1]$ :

$$
\begin{aligned}
\|s[k+1]\|_{\infty} & \leq\|I+T Q(\alpha(k T))\|_{\infty}\|s[k]\|_{\infty}+T\left\|R B(\alpha(k T)) e_{u}[k]\right\|_{\infty}+\left\|R e_{p}[k]\right\|_{\infty}+\left\|e_{z 2}[k]\right\|_{\infty} \\
& \leq\left(1+\lambda_{2} T\right)\|s[k]\|_{\infty}+T\|R B(\alpha(k T))\|\left\|e_{u}[k]\right\|_{\infty}+\|R\|\left\|e_{p}[k]\right\|_{\infty}+\left\|e_{z 2}[k]\right\|_{\infty},
\end{aligned}
$$

and using (54), (55), and (56), there exist constants $e_{3}>0$ and $w_{3}>0$ such that for small $T$

$$
\begin{aligned}
\|s[k+1]\|_{\infty} \leq & \left(1+\lambda_{2} T+e_{3} T(\bar{\delta}+T)\right)\|s[k]\|_{\infty}+e_{3} T(\bar{\delta}+T)\left\|\left[\begin{array}{l}
\bar{x}[k]] \\
\bar{z}[k]
\end{array}\right]\right\|_{1}+e_{3} T^{2} v[k] \\
& +w_{3} T\left\|w_{n}\right\|_{\infty}+w_{3} T\left\|w_{d}\right\|_{\infty} .
\end{aligned}
$$

Now we combine the bounds on the states. Recall that the controller parameter $\rho$ was chosen to satisfy $\rho \in(0,-\lambda)$, which means that $\lambda+\rho<0$; now fix

$$
\bar{\lambda} \in\left(\max \left\{\lambda_{1}, \lambda_{2}, \lambda+\rho\right\}, 0\right),
$$


which means that

$$
\lambda_{2}<\lambda_{1}<\bar{\lambda}<0 .
$$

Combining the bounds given in (52), (57), and (58) yields, for small $T$ :

$$
\begin{aligned}
& {\left[\begin{array}{c}
v[k+1] \\
\left\|\left[\begin{array}{c}
\bar{x}[k+1] \\
\bar{z}[k+1]
\end{array}\right]\right\|_{1}\|s[k+1]\|_{\infty}
\end{array}\right] \leq\left[\begin{array}{ccc}
1+\bar{\lambda} T+e_{1} T^{2} & \gamma_{1} T & \gamma_{2} T \\
e_{2} T^{2} & 1+\lambda_{1} T+e_{2} T(\bar{\delta}+T) & \gamma_{3} T+e_{2} T(\bar{\delta}+T) \\
e_{3} T^{2} & e_{3} T(\bar{\delta}+T) & 1+\lambda_{2} T+e_{3} T(\bar{\delta}+T)
\end{array}\right]\left[\begin{array}{c}
v[k] \\
\|[\bar{x}[k]] \\
\bar{z}[k] \\
\|s[k]\|_{\infty}
\end{array}\right]} \\
& +\left[\begin{array}{cc}
w_{1} T & w_{1} T^{2} \\
0 & w_{2} T \\
w_{3} T & w_{3} T
\end{array}\right]\left[\begin{array}{l}
\left\|w_{n}\right\|_{\infty} \\
\left\|w_{d}\right\|_{\infty}
\end{array}\right] .
\end{aligned}
$$

So, with $E \in \mathbb{R}^{3 \times 3}$ suitably chosen and $T$ sufficiently small, we have

$$
\begin{aligned}
{\left[\begin{array}{c}
v[k+1] \\
\left.\left\|\left[\begin{array}{c}
\bar{x}[k+1] \\
\bar{z}[k+1]
\end{array}\right]\right\|_{1}\right]\|s[k+1]\|_{\infty}
\end{array}\right] \leq } & (\underbrace{\left[\begin{array}{ccc}
1+\bar{\lambda} T & \gamma_{1} T & \gamma_{2} T \\
0 & 1+\lambda_{1} T & \gamma_{3} T \\
0 & 0 & 1+\lambda_{2} T
\end{array}\right]}_{=: \Lambda}+T(\bar{\delta}+T) E)\left[\begin{array}{c}
v[k] \\
\left\|\left[\begin{array}{l}
\bar{x}[k] \\
\bar{z}[k]
\end{array}\right]\right\|_{1} \\
\|s[k]\|_{\infty}
\end{array}\right] \\
& +\underbrace{\left[\begin{array}{cc}
w_{1} T & w_{1} T^{2} \\
0 & w_{2} T \\
w_{3} T & w_{3} T
\end{array}\right]}_{=: W(T)}\left[\begin{array}{l}
\left\|w_{n}\right\|_{\infty} \\
\left\|w_{d}\right\|_{\infty}
\end{array}\right] .
\end{aligned}
$$

Next we define three states as upper bounds of the above states at periods $k$ and $k+1$. This allows us to get equality, so we can solve and transform a difference equation rather than inequality. Define $\psi:=\left(\psi_{1}, \psi_{2}, \psi_{3}\right) \in \mathbb{R}_{+}^{3}$ via

$$
\begin{gathered}
\psi_{1}[k]:=v[k], \quad \psi_{2}[k]:=\left\|\left[\begin{array}{l}
\bar{x}[k] \\
\bar{z}[k]
\end{array}\right]\right\|_{1}, \quad \psi_{3}[k]:=\|s[k]\|_{\infty}, \\
\psi[k+1]:=(\Lambda+T(\bar{\delta}+T) E) \psi[k]+W(T)\left[\begin{array}{l}
\left\|w_{n}\right\|_{\infty} \\
\left\|w_{d}\right\|_{\infty}
\end{array}\right] .
\end{gathered}
$$

It is clear that $v[k+1] \leq \psi_{1}[k+1],\left\|\left[\begin{array}{l}\bar{x}[k+1] \\ \bar{z}[k+1]\end{array}\right]\right\|_{1} \leq \psi_{2}[k+1]$, and $\|s[k+1]\|_{\infty} \leq \psi_{3}[k+1]$ because

$$
\begin{aligned}
& {\left[\begin{array}{c}
v[k+1] \\
\left\|\left[\begin{array}{c}
\bar{x}[k+1] \\
\bar{z}[k+1]
\end{array}\right]\right\|_{1} \\
\|s[k+1]\|_{\infty}
\end{array}\right] \leq(\Lambda+T(\bar{\delta}+T) E)\left[\begin{array}{c}
v[k] \\
\|\left[\begin{array}{c}
\bar{x}[k] \\
\bar{z}[k] \\
\|s[k]\|_{\infty}
\end{array}\right]
\end{array}\right]+W(T)\left[\begin{array}{c}
\left\|w_{n}\right\|_{\infty} \\
\left\|w_{d}\right\|_{\infty}
\end{array}\right]} \\
& =(\Lambda+T(\bar{\delta}+T) E) \psi[k]+W(T)\left[\begin{array}{l}
\left\|w_{n}\right\|_{\infty} \\
\left\|w_{d}\right\|_{\infty}
\end{array}\right] \\
& =\psi[k+1] .
\end{aligned}
$$

Next we perform two similarity transformations with the objective of diagonalizing the matrix $\Lambda$ in (60). The first is a constant transformation of the form 


$$
V:=\left[\begin{array}{ccc}
1 & 0 & 0 \\
0 & 1 & v_{23} \\
0 & 0 & 1
\end{array}\right]
$$

so that under similiarity transformation we have

$$
V(\Lambda+T(\bar{\delta}+T) E) V^{-1}=\left[\begin{array}{ccc}
1+\bar{\lambda} T & \gamma_{1} T & \left(\gamma_{2}-v_{23} \gamma_{1}\right) T \\
0 & 1+\lambda_{1} T & \left(\gamma_{3}+v_{23}\left(\lambda_{2}-\lambda_{1}\right)\right) T \\
0 & 0 & 1+\lambda_{2} T
\end{array}\right]+T(\bar{\delta}+T) V E V^{-1} .
$$

Choose $v_{23}=\gamma_{3} /\left(\lambda_{1}-\lambda_{2}\right)$ so that we are left with

$$
V(\Lambda+T(\bar{\delta}+T) E) V^{-1}=\left[\begin{array}{ccc}
1+\bar{\lambda} T & \gamma_{1} T & \left(\gamma_{2}-v_{23} \gamma_{1}\right) T \\
0 & 1+\lambda_{1} T & 0 \\
0 & 0 & 1+\lambda_{2} T
\end{array}\right]+T(\bar{\delta}+T) V E V^{-1} .
$$

To complete the diagonalization of $\Lambda$ consider a transformation of the form

$$
Y:=\left[\begin{array}{cc}
1 & \bar{Y} \\
0 & I
\end{array}\right] \in \mathbb{R}^{3 \times 3}, \quad \bar{Y} \in \mathbb{R}^{1 \times 2},
$$

so that, with $\gamma_{4}:=\gamma_{2}-v_{23} \gamma_{1}$,

$$
\begin{aligned}
& Y V(\Lambda+T(\bar{\delta}+T) E) V^{-1} Y^{-1}=\left[\begin{array}{cc}
1+\bar{\lambda} T & {\left[\begin{array}{ll}
\gamma_{1} & \gamma_{4}
\end{array}\right] T+\bar{Y}\left[\begin{array}{cc}
1+\lambda_{1} T & 0 \\
0 & 1+\lambda_{2} T
\end{array}\right]-(1+\bar{\lambda} T) \bar{Y}} \\
{\left[\begin{array}{l}
0 \\
0
\end{array}\right]} & {\left[\begin{array}{cc}
1+\lambda_{1} T & 0 \\
0 & 1+\lambda_{2} T
\end{array}\right]}
\end{array}\right] \\
& +T(\bar{\delta}+T) Y V E V^{-1} Y^{-1} .
\end{aligned}
$$

Choose $\bar{Y}=\left[\begin{array}{ll}\frac{\gamma_{1}}{\lambda-\lambda_{1}} & \frac{\gamma_{4}}{\lambda-\lambda_{2}}\end{array}\right]$ to get

$$
Y V(\Lambda+T(\bar{\delta}+T) E) V^{-1} Y^{-1}=\left[\begin{array}{ccc}
1+\bar{\lambda} T & 0 & 0 \\
0 & 1+\lambda_{1} T & 0 \\
0 & 0 & 1+\lambda_{2} T
\end{array}\right]+T(\bar{\delta}+T) Y V E V^{-1} Y^{-1} .
$$

Defining $N:=Y V$, we have

$$
N \psi[k+1]=\left(\left[\begin{array}{ccc}
1+\bar{\lambda} T & 0 & 0 \\
0 & 1+\lambda_{1} T & 0 \\
0 & 0 & 1+\lambda_{2} T
\end{array}\right]+T(\bar{\delta}+T) N E N^{-1}\right) N \psi[k]+N W(T)\left[\begin{array}{l}
\left\|w_{n}\right\|_{\infty} \\
\left\|w_{d}\right\|_{\infty}
\end{array}\right] .
$$

It follows from (59) that all elements of $N$ are non-negative, which can be used to prove that

$$
\|N \psi[k+1]\| \geq\left\|N\left[\begin{array}{c}
v[k+1] \\
\left\|\left[\begin{array}{c}
\bar{x}[k+1] \\
\bar{z}[k+1]
\end{array}\right]\right\|_{1} \\
\|s[k+1]\|_{\infty}
\end{array}\right]\right\|=\|p[k+1]\| .
$$


Taking the $\infty$-norm of $p[k+1]$, there exist constants $\gamma>0$ and $c>0$ such that for small $T$

$$
\begin{aligned}
\|p[k+1]\|_{\infty} & \leq\|N \psi[k+1]\|_{\infty} \leq(1+\bar{\lambda} T+\gamma T(\bar{\delta}+T))\|N \psi[k]\|_{\infty}+c T\left\|w_{n}\right\|_{\infty}+c T\left\|w_{d}\right\|_{\infty} \\
& =(1+\bar{\lambda} T+\gamma T(\bar{\delta}+T))\|p[k]\|_{\infty}+c T\left\|w_{n}\right\|_{\infty}+c T\left\|w_{d}\right\|_{\infty} .
\end{aligned}
$$

Now choose $\bar{\delta}>0$ so that

$$
\hat{\lambda}:=\bar{\lambda}+2 \gamma \bar{\delta}<0
$$

it follows that for small $T$, we have

$$
\begin{aligned}
\|p[k+1]\|_{\infty} & \leq(1+\hat{\lambda} T)\|p[k]\|_{\infty}+c T\left\|w_{n}\right\|_{\infty}+c T\left\|w_{d}\right\|_{\infty} \\
& \leq \mathrm{e}^{\hat{\lambda} T}\|p[k]\|_{\infty}+c T\left\|w_{n}\right\|_{\infty}+c T\left\|w_{d}\right\|_{\infty},
\end{aligned}
$$

which means that (iii) holds.

\section{Proof of (i)}

By the definition of $p$,

$$
p(t)-p[k]=N\left[\begin{array}{c}
|v(t)|-|v[k]| \\
\left\|\left[\begin{array}{c}
\bar{x}(t) \\
\bar{z}(t)
\end{array}\right]\right\|_{1}-\left\|\left[\begin{array}{c}
\bar{x}[k] \\
\bar{z}[k]
\end{array}\right]\right\|_{1} \\
\|s(t)\|_{\infty}-\|s[k]\|_{\infty}
\end{array}\right], \quad t \geq 0 .
$$

Taking the 1-norm and using the reverse triangle inequality, for $t \in[k T,(k+1) T)$ we get

$$
\begin{aligned}
\|p(t)-p[k]\|_{1}= & \mathcal{O}(1)|v(t)-v[k]|+\mathcal{O}(1)\|x(t)-x[k]\| \\
& +\mathcal{O}(1)\|z(t)-z[k]\|+\mathcal{O}(1)\|r(t)-r[k]\| .
\end{aligned}
$$

The solution to (15) with initial condition $v[k]$ is

$$
v(t)=v[k]+\int_{k T}^{t} \lambda(v(\tau)-v[k]) \mathrm{d} \tau+(t-k T) \lambda v[k]+\int_{k T}^{t}(\|u(\tau)\|+\|y(\tau)\|) \mathrm{d} \tau .
$$

Rearranging and taking the absolute value, we have

$$
|v(t)-v[k]| \leq|\lambda| \int_{k T}^{t}|v(\tau)-v[k]| \mathrm{d} \tau+T|\lambda| v[k]+\int_{k T}^{t}(\|u(\tau)\|+\|y(\tau)\|) \mathrm{d} \tau,
$$

and by applying the Bellman-Gronwall inequality and using Proposition 3, it follows that

$$
\begin{aligned}
|v(t)-v[k]|= & \mathcal{O}(T) v[k]+\mathcal{O}(T)\left\|\left[\begin{array}{c}
\bar{x}[k]] \\
\bar{z}[k]
\end{array}\right]\right\|_{1}+\mathcal{O}(T)\|s[k]\|_{\infty} \\
& +\mathcal{O}(T)\left\|w_{n}\right\|_{\infty}+\mathcal{O}\left(T^{2}\right)\left\|w_{d}\right\|_{\infty}, \quad t \in[k T,(k+1) T) .
\end{aligned}
$$

For $t \in[k T,(k+1) T)$ we also have $\|z(t)-z[k]\|=0$ and $\|r(t)-r[k]\|=0$. Using these bounds on (61), along with Proposition 3 and (62), we get

$$
\|p(t)-p[k]\|=\mathcal{O}(T)\|p[k]\|+\mathcal{O}(T)\left(\left\|w_{n}\right\|_{\infty}+\left\|w_{d}\right\|_{\infty}\right), \quad t \in[k T,(k+1) T) .
$$


So, for $t \in[k T,(k+1) T)$, there exists a constant $c>0$ so that for sufficiently small $T$.

$$
\|p(t)-p[k]\| \leq c T\|p[k]\|+c T\left\|w_{n}\right\|_{\infty}+c T\left\|w_{d}\right\|_{\infty}, \quad t \in[k T,(k+1) T) .
$$

\section{Proof of (ii)}

The bound (61) derived in the proof of part (i) remains valid. Additionally, using (17) we have

$$
z[k+1]-z[k]=T F(\hat{\alpha}[k]) z[k]+T G(\hat{\alpha}[k]) r[k],
$$

so taking the norm and employing order notation,

$$
\|z[k+1]-z[k]\|=\mathcal{O}(T)\left\|\left[\begin{array}{l}
\bar{x}[k] \\
\bar{z}[k]
\end{array}\right]\right\|_{1}+\mathcal{O}(T)\|s[k]\|_{\infty}=\mathcal{O}(T)\|p[k]\| .
$$

We can also upper bound $\|r[k+1]-r[k]\|$. We have, again from (17),

$$
\begin{aligned}
r[k+1]-r[k]= & T R B(\hat{\alpha}[k]) H(\hat{\alpha}[k]) z[k]+T(Q(\hat{\alpha}[k])+R B(\hat{\alpha}[k]) K(\hat{\alpha}[k]) M) r[k] \\
& -T L(\hat{\alpha}[k])\left(C(\alpha[k]) x[k]+w_{n}[k]\right),
\end{aligned}
$$

so taking the norm and employing order notation,

$$
\|r[k+1]-r[k]\|=\mathcal{O}(T)\|p[k]\|+\mathcal{O}(T)\left\|w_{n}\right\|_{\infty} .
$$

Applying these bounds to (61), along with Proposition 3 and (52), we have

$$
\|p[k+1]-p[k]\|=\mathcal{O}(T)\|p[k]\|+\mathcal{O}(T)\left\|w_{n}\right\|_{\infty}+\mathcal{O}(T)\left\|w_{d}\right\|_{\infty} .
$$

So there exists a constant $c>0$ so that, for all $T$ sufficiently small,

$$
\|p[k+1]\| \leq(1+c T)\|p[k]\|+c T\left\|w_{n}\right\|_{\infty}+c T\left\|w_{d}\right\|_{\infty} .
$$

\section{References}

[1] G. C. Goodwin, P. J. Ramadge, and P. E. Caines. Discrete-time multivariable adaptive control. IEEE Trans. Autom. Control, 25(3):449-456, June 1980. 2

[2] A. S. Morse. Global stability of parameter-adaptive control systems. IEEE Trans. Autom. Control, 25(3):433-439, June 1980. 2

[3] K. S. Narendra, Y. H. Lin, and L. S. Valavani. Stable adaptive controller design, part ii: Proof of stability. IEEE Trans. Autom. Control, 25(3):440-448, June 1980. 2

[4] C. E. Rohrs, L. Valavani, M. Athans, and G. Stein. Robustness of adaptive control algorithms in the presence of unmodeled dynamics. IEEE Trans. Autom. Control, 30(9):881-889, September 1985. 2 
[5] P. A. Ioannou and J. Sun. Robust Adaptive Control. Prentice Hall, 1996. 2

[6] C. I. Byrnes and J. C. Willems. Adaptive stabilization of multivariable linear systems. The 23rd IEEE Conference on Decision and Control, 23:1574-1577, December 1984. 2

[7] D. E. Miller and E. J. Davison. An adaptive controller which provides arbitrarily good transient and steady-state response. IEEE Trans. Autom. Control, 36(1):68-81, January 1991. 2

[8] A. S. Morse. Supervisory control of families of linear set-point controllers - part 1: Exact matching. IEEE Trans. Autom. Control, 41(10):1413-1431, October 1996. 2

[9] A. S. Morse. Supervisory control of families of linear set-point controllers - part 2: Robustness. IEEE Trans. Autom. Control, 42(11):1500-1515, November 1997. 2

[10] K. S. Narendra and J. Balakrishnan. Adaptive control using multiple models. IEEE Trans. Autom. Control, 42(2):171-187, February 1997. 2

[11] J. Hocherman-Frommer, S. R. Kulkarni, and P. J. Ramadge. Controller switching based on output prediction errors. IEEE Trans. Autom. Control, 43(5):596-607, May 1998. 2

[12] J. P. Hespanha, D. Liberzon, and A. S. Morse. Overcoming the limitations of adaptive control by means of logic based switching. Systems and Control Letters, 49(1):49-65, May 2003. 2

[13] G. Kreisselmeir. Adaptive control of a class of slowly time-varying plants. Systems and Control Letters, 8(2):97-103, December 1986. 2

[14] R. H. Middleton and G. C. Goodwin. Adaptive control of time-varying linear systems. IEEE Trans. Autom. Control, 33(2):150-155, February 1988. 2

[15] K. S. Tsakalis and P. A. Ioannou. Adaptive control of linear time-varying plants: A new model reference controller structure. IEEE Trans. Autom. Control, 34(10):1038-1046, October 1989. 2

[16] P. G. Voulgaris, M. A. Dahleh, and L. S. Valavani. Robust adaptive control: a slowly varying systems approach. Automatica, 30(9):1455-1461, September 1994. 2

[17] D. Dimogianopoulos and R. Lozano. Adaptive control for linear slowly time-varying systems using direct least-squares estimation. Automatica, 37(2):251-256, February 2001. 2

[18] B. Fidan, Y. Zhang, and P. Ioannou. Adaptive control of a class of slowly time-varying systems with modelling uncertainties. IEEE Trans. Autom. Control, 50(6):915-920, June 2005. 2

[19] L. Vu and D. Liberzon. Supervisory control of uncertain linear time-varying systems. IEEE Trans. Autom. Control, 56(1):27-42, January 2011. 2

[20] J. R. Vale and D. E. Miller. Step tracking in the presence of persistent plant changes. IEEE Trans. Autom. Control, 56(1):43-58, January 2011. 2

[21] D. E. Miller and J. R. Vale. Pole placement adaptive control with persistent jumps in the plant parameters. Mathematics of Control, Signals, and Systems, 16:177-214, June 2014. 2 
[22] K. S. Tsakalis and P. A. Ioannou. A new indirect adaptive control scheme for time-varying plants. IEEE Trans. Autom. Control, 35(6):697-705, June 1990. 2

[23] Z. Tian and K. S. Narendra. Adaptive control of linear periodic systems. 2009 American Control Conference, June 2009. 2

[24] A. M. Annaswamy and K. S. Narendra. Adaptive control of a first order plant with a timevarying parameter. American Control Conference, pages 975-980, June 1989. 2, 5

[25] D. E. Miller. A new approach to model reference adaptive control. IEEE Trans. Autom. Control, 48(5):743-757, May 2003. 2, 5, 7

[26] A. Ilchmann, E. P. Ryan, and S. Trenn. Tracking control: Performance funnels and prescribed transient behaviour. Systems and Control Letters, 54(7):655-670, July 2005. 2, 5, 7

[27] C. M. Hackl, N. Hopfe, A. Ilchmann, M. Mueller, and S. Trenn. Funnel control for systems with relative degree two. SIAM Journal of Control and Optimization, 51(2):965-995, 2013. $2,5,7$

[28] R. Marino and P. Tomei. Adaptive control of linear time-varying systems. Automatica, 39(4):651-659, April 2003. 2, 5, 7

[29] D. E. Miller and N. Mansouri. Model reference adaptive control using simultaneous probing, estimation, and control. IEEE Trans. Autom. Control, 55(9):2014-2029, September 2010. 2, 5,7

[30] V. Rudko and D. Miller. Nonlinear periodic adaptive control for linear time-varying plants. American Control Conference, 2016. 2, 3

[31] W. J. Rugh. Analytical framework for gain scheduling. IEEE Control Systems, 11(1):79-84, June 1991. 2

[32] J. S. Shamma and M. Athans. Analysis of gain scheduled control for nonlinear plants. IEEE Trans. Autom. Control, 35(8):898-907, August 1990. 2

[33] W. J. Rugh and J. S. Shamma. Research on gain scheduling. Automatica, 36:1401-1425, 2000. 2

[34] F. Blanchini. Ultimate boundedness control for uncertain discrete-time systems via setinduced lyapunov functions. Proceeding of the 30th IEEE Conference on Decision and Contro, 2:1755-1760, December 1991. 2

[35] F. Blanchini. Nonquadratic lyapunov functions for robust control. Automatica, 31(3):451-461, March 1995. 2

[36] F. Blanchini. The gain scheduling and the robust state feedback stabilization problems. IEEE Trans. Autom. Control, 45(11):2061-2070, November 2000. 2

[37] F. Blanchini, S. Miani, and C. Savorgnan. Stability results for linear parameter varying and switching systems. Automatica, 43(10):1817-1823, October 2007. 2 
[38] F. Blanchini, D. Casagrande, S. Miani, and U. Viaro. Stable LPV realization of parametric transfer functions and its application to gain-scheduling control design. IEEE Trans. Autom. Control, 55(10):2271-2281, October 2010. 2, 3, 5, 6, 13, 23

[39] D. E. Miller. Near optimal lqr performance for a compact set of plants. IEEE Trans. Autom. Control, 51(9):1423-1439, September 2006. 3, 8

[40] V. Rudko. Nonlinear Period Adaptive Control for Linear Time-Varying Plants. Master's thesis, University of Waterloo, Canada, 2013. Available: https://uwspace.uwaterloo.ca/handle/10012/7775. 6, 10, 25

[41] M. Vidyasagar. Control System Synthesis: A Factorization Approach. The MIT Press, 1985. 21 